Supporting Information..........

\title{
The HETTAP Approach: Self-Assembly and Metal Ion Sensing of Dumbbell-Shaped Molecules and Clip Molecules
}

\author{
Michael Schmittel, ${ }^{a}{ }^{a}$ Venkateshwarlu Kalsani, ${ }^{a}$ Prasenjit Mal, ${ }^{a}$ Jan W. Bats ${ }^{b}$
}

${ }^{a}$ Center of Micro and Nanochemistry and Engineering, Organische Chemie I, Universität Siegen, Adolf-Reichwein-Str. 2, D-57068 Siegen, Germany, Fax: (+49) 271740 3270; E-mail: schmittel@chemie.uni-siegen.de

${ }^{b}$ Institut für Organische Chemie und Chemische Biologie, Johann Wolfgang GoetheUniversität, Marie-Curie-Strasse 11, D-60439 Frankfurt am Main, Germany.
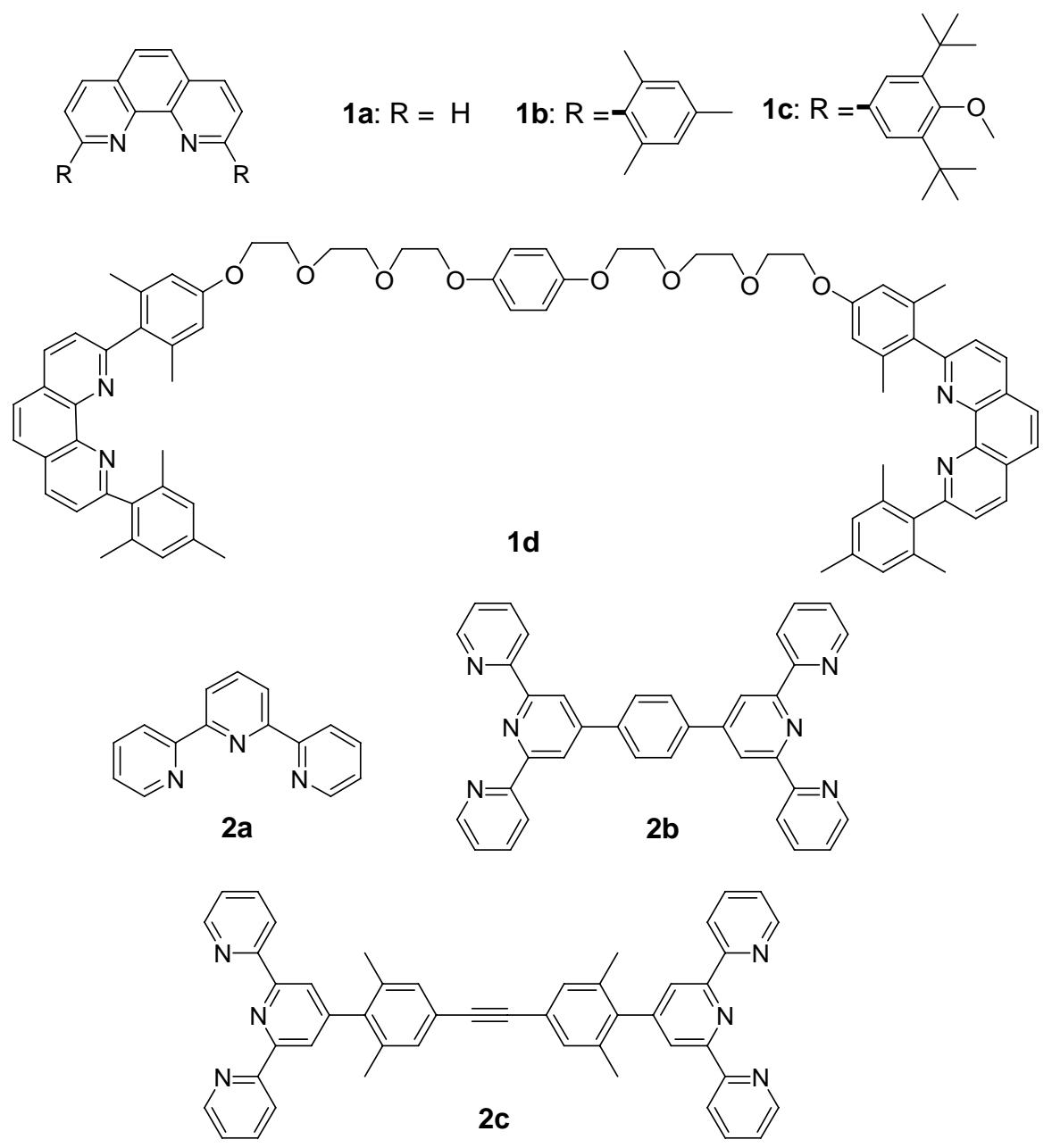
Table S1: Crystal data and structure refinement for $\left[\mathrm{Zn}_{2}(\mathbf{1 b})_{2}(\mathbf{2 b})\right]^{4+}$.

\begin{tabular}{|c|c|}
\hline Substance & {$\left[\mathrm{Zn}_{2}(\mathbf{1 b})_{2}(\mathbf{2 b})\right]^{4+}$} \\
\hline Sum formula & $\mathrm{C}_{100} \mathrm{H}_{80} \mathrm{~F}_{12} \mathrm{~N}_{10} \mathrm{O}_{12} \mathrm{~S}_{4} \mathrm{Zn}_{2}$ \\
\hline Formula weight $/ \mathrm{g} \cdot \mathrm{mol}^{-1}$ & 2100.72 \\
\hline Temperature /K & $156(2)$ \\
\hline Wavelength /pm & 71.073 \\
\hline Crystal system, space group & Triclinic, $P-1$ \\
\hline \multirow[t]{4}{*}{ Unit cell dimensions } & $\mathrm{a}=11.521(2) \AA$ \\
\hline & $\mathrm{b}=17.185$ (4) $\AA$ \\
\hline & $c=18.164(4) \AA$ \\
\hline & $\beta=94.469$ (19) ${ }^{\circ}$ \\
\hline Volume $/ \AA^{3}$ & $3074.0(11)$ \\
\hline Z, Calculated density $/ \mathrm{Mg} \cdot \mathrm{m}^{-3}$ & $1,1.135$ \\
\hline Absorption coefficient $/ \mathrm{mm}^{-1}$ & 0.529 \\
\hline $\mathrm{F}(000)$ & 1078 \\
\hline Crystal size $/ \mathrm{mm}^{3}$ & $0.36 \times 0.32 \times 0.10$ \\
\hline Reflections collected / unique / $\mathrm{R}_{\text {int }}$ & 30425 / 11341 / 0.1577 \\
\hline Refinement method & Full-matrix least-squares on $F^{2}$ \\
\hline Data / restraints / parameters & 11341 / 4 / 637 \\
\hline Goodness-of-fit on $F^{2}$ & 0.988 \\
\hline Final $R$ indices $[\mathrm{I}>2 \sigma(\mathrm{I})]$ & $R 1=0.1166, w R 2=0.2564$ \\
\hline $\mathrm{R}$ indices (all data) & $R 1=0.2382, w R 2=0.2930$ \\
\hline Largest diff. peak and hole & 1.069 and $-0.690 \mathrm{e} / \AA^{3}$ \\
\hline
\end{tabular}


Table S2: Atomic coordinates ( x $10^{5}$ ) and equivalent isotropic displacement parameters $\left(\AA^{2} \mathrm{x}\right.$ $\left.10^{4}\right)$ for $\left[Z_{2}(\mathbf{1 b})_{2}(\mathbf{2 b})\right]^{4+} . U(\mathrm{eq})$ is defined as one third of the trace of the orthogonalized $U \mathrm{ij}$ tensor.

\begin{tabular}{|c|c|c|c|c|}
\hline Atom & $\bar{x}$ & $\bar{y}$ & $\bar{z}$ & $\mathbf{U}_{\text {eq }}$ \\
\hline $\mathrm{Zn}$ & 74098(10) & $48050(7)$ & $24279(7)$ & $221(4)$ \\
\hline $\mathrm{S}(1)$ & $82420(30)$ & 78600(30) & $66330(20)$ & $677(11)$ \\
\hline$S(2)$ & $73420(60)$ & $121320(40)$ & $90460(40)$ & $1480(20)$ \\
\hline $\mathrm{N}(1)$ & $80200(60)$ & $61710(50)$ & $30450(40)$ & $114(16)$ \\
\hline $\mathrm{N}(3)$ & $89340(60)$ & 51580(50) & $19000(40)$ & 217(18) \\
\hline $\mathrm{N}(2)$ & $61640(70)$ & 51220(50) & $32920(50)$ & $270(20)$ \\
\hline $\mathrm{N}(5)$ & $77200(60)$ & 37230(50) & $25110(50)$ & 212(18) \\
\hline$C(45)$ & $89090(90)$ & 50950(70) & $42470(60)$ & $300(30)$ \\
\hline $\mathrm{N}(4)$ & $60930(70)$ & 37880(60) & $14660(50)$ & $320(20)$ \\
\hline C(18) & $93670(90)$ & $46040(70)$ & $12920(60)$ & $390(30)$ \\
\hline$C(36)$ & $63360(80)$ & 50930(70) & $7790(50)$ & $300(30)$ \\
\hline $\mathrm{C}(31)$ & $54770(90)$ & $48270(60)$ & $11030(60)$ & $300(30)$ \\
\hline$C(14)$ & $94790(90)$ & 60400(70) & $22250(60)$ & $340(30)$ \\
\hline$C(7)$ & 89990(80) & 66540(60) & $29100(60)$ & $240(20)$ \\
\hline$C(15)$ & 105140(90) & 64340(70) & 19370(60) & $380(30)$ \\
\hline $\mathrm{O}(1)$ & $79990(80)$ & 69290(60) & $62480(60)$ & $860(30)$ \\
\hline$C(12)$ & $44450(90)$ & 47770(70) & $39040(60)$ & $340(30)$ \\
\hline$C(40)$ & $94910(90)$ & 46990(70) & $36560(70)$ & $440(30)$ \\
\hline$C(5)$ & 79340(90) & 75180(70) & $41420(60)$ & $400(30)$ \\
\hline $\mathrm{C}(6)$ & $74580(80)$ & $66060(60)$ & $36600(60)$ & 280(20) \\
\hline C(19) & 59840(90) & $29280(60)$ & $13580(60)$ & $280(20)$ \\
\hline $\mathrm{O}(2)$ & $95590(80)$ & 83930(60) & $68280(70)$ & $1090(40$ \\
\hline $\mathrm{C}(3)$ & $95260(100)$ & $90250(80)$ & $45090(70)$ & $520(30)$ \\
\hline $\mathrm{C}(30)$ & $69290(100)$ & 28980(70) & 19380(50) & $360(30)$ \\
\hline $\mathrm{C}(9)$ & $64070(90)$ & 60070(60) & $37430(60)$ & $260(20)$ \\
\hline $\mathrm{C}(8)$ & $95030(80)$ & 75820(60) & $33650(60)$ & $340(30)$ \\
\hline C(32) & 48380(90) & 53630(70) & $16050(60)$ & $360(30)$ \\
\hline $\mathrm{C}(17)$ & $103340(100)$ & 48970(80) & $9730(70)$ & $550(30)$ \\
\hline $\mathrm{C}(1)$ & $104950(120)$ & $105140(80)$ & $46150(80)$ & $750(40)$ \\
\hline $\mathrm{C}(26)$ & $69400(120)$ & $20630(70)$ & 18870(70) & $520(30)$ \\
\hline$C(20)$ & $52370(100)$ & $38540(60)$ & $9500(60)$ & $390(30)$ \\
\hline $\mathrm{C}(11)$ & 47200(100) & 56640(90) & $43580(70)$ & $510(30)$ \\
\hline C(33) & $52440(100)$ & 62820(70) & $18800(60)$ & $410(30)$ \\
\hline$C(4)$ & $89750(90)$ & 80230(60) & $39670(70)$ & $420(30)$ \\
\hline C(29) & $86040(90)$ & 37340(70) & $30320(60)$ & $370(30)$ \\
\hline$C(28)$ & 87340(100) & 28860(80) & 29870(70) & $450(30)$ \\
\hline$C(48)$ & $79430(100)$ & $48070(70)$ & $45570(60)$ & $530(30)$ \\
\hline$C(34)$ & $62830(110)$ & 66720(70) & $16160(70)$ & $460(30)$ \\
\hline $\mathrm{C}(2)$ & $100220(110)$ & $95860(70)$ & $41620(70)$ & $540(30)$ \\
\hline$C(16)$ & 109110(100) & 58310(80) & 13370(70) & $530(30)$ \\
\hline $\mathrm{F}(3)$ & 81920(110) & $90100(70)$ & $81360(60)$ & $1670(60)$ \\
\hline
\end{tabular}




\begin{tabular}{|c|c|c|c|c|}
\hline $\mathrm{C}(13)$ & $52680(100)$ & $45440(70)$ & $33740(60)$ & $390(30)$ \\
\hline $\mathrm{O}(3)$ & 74550(90) & $81870(80)$ & $62670(60)$ & $1050(30)$ \\
\hline C(22) & $42470(120)$ & 22060(80) & $2800(80)$ & $680(40)$ \\
\hline C(43) & $107010(170)$ & $65630(120)$ & $45830(120)$ & $910(60)$ \\
\hline $\mathrm{C}(41)$ & $105960(100)$ & $50340(110)$ & $34590(80)$ & $660(40)$ \\
\hline C(39) & 69920(100) & $46500(80)$ & $1510(60)$ & $580(40)$ \\
\hline C(35) & $67850(100)$ & 61690(70) & $11170(60)$ & $440(30)$ \\
\hline C(10) & 57050(90) & $63420(80)$ & 43370(70) & $500(30)$ \\
\hline $\mathrm{C}(21)$ & 42510(120) & 30840(90) & $3780(70)$ & $680(40)$ \\
\hline C(23) & $51220(110)$ & $21430(70)$ & $8110(70)$ & $440(30)$ \\
\hline C(25) & $60230(130)$ & 12590(80) & $12460(70)$ & $590(40)$ \\
\hline C(37) & $37600(100)$ & $49130(80)$ & 18990(70) & $640(40)$ \\
\hline $\mathrm{O}(5)$ & $75510(100)$ & 125200(80) & $99860(70)$ & $1660(60)$ \\
\hline $\mathrm{O}(6)$ & $86600(130)$ & $121410(100)$ & 88330(100) & $1900(60)$ \\
\hline$C(44)$ & $96680(150)$ & $61710(100)$ & $47690(80)$ & $790(40)$ \\
\hline $\mathrm{F}(4)$ & $53480(120)$ & $108200(70)$ & 87910(80) & $1940(60)$ \\
\hline $\mathrm{C}(42)$ & $111760(120)$ & 59940(130) & 39550(110) & $870(60)$ \\
\hline$C(46)$ & $110920(120)$ & $44160(110)$ & 27660(90) & $1080(60)$ \\
\hline $\mathrm{C}(27)$ & 78930(120) & 20500(90) & 24210(80) & $620(40)$ \\
\hline C(49) & $79200(200)$ & 80730(180) & $76520(120)$ & $1220(70)$ \\
\hline $\mathrm{F}(1)$ & $68860(120)$ & $76210(70)$ & $76340(70)$ & $1950(70)$ \\
\hline $\mathrm{C}(38)$ & $67470(120)$ & 77360(90) & 19490(80) & $920(50)$ \\
\hline $\mathrm{O}(4)$ & $63780(150)$ & $122130(140)$ & $85770(140)$ & $3060(150)$ \\
\hline$C(24)$ & 50930(130) & 12760(90) & $7550(90)$ & $700(40)$ \\
\hline $\mathrm{F}(2)$ & 87380(130) & $78760(80)$ & 80690(70) & $1650(50)$ \\
\hline$F(6)$ & $64710(120)$ & 104210(80) & $79500(70)$ & $2100(60)$ \\
\hline$C(50)$ & $66870(180)$ & $109100(120)$ & 88290(120) & $2600(200)$ \\
\hline$C(47)$ & $115000(150)$ & $75020(110)$ & $50440(110)$ & $1320(70)$ \\
\hline $\mathrm{F}(5)$ & $72100(300)$ & $106800(300)$ & $92100(300)$ & $6400(400)$ \\
\hline
\end{tabular}


Table S3: Anisotropic displacement parameters $\left(\AA^{2} \times 10^{4}\right)$ for $\left[\mathrm{Zn}_{2}(\mathbf{1 b})_{2}(\mathbf{2 b})\right]^{4+}$. The anisotropic displacement factor exponent takes the form: $\left\{-2 \pi^{2}\left[\mathrm{~h}^{2} \mathrm{a}^{* 2} U_{11}+\ldots+2 h k a^{*} b^{*} U_{12}\right]\right.$

\begin{tabular}{|c|c|c|c|c|c|c|}
\hline Atom & $\boldsymbol{U}_{11}$ & $\boldsymbol{U}_{22}$ & $\boldsymbol{U}_{33}$ & $\boldsymbol{U}_{23}$ & $\boldsymbol{U}_{13}$ & $\boldsymbol{U}_{12}$ \\
\hline $\mathrm{Zn}$ & $210(6)$ & 261(7) & 271(7) & 163(6) & $128(5)$ & 111(5) \\
\hline $\mathrm{S}(1)$ & $600(20)$ & $620(30)$ & $720(30)$ & $310(20)$ & $200(20)$ & $50(20)$ \\
\hline $\mathrm{S}(2)$ & $1160(50)$ & $1110(40)$ & $1620(60)$ & $180(40)$ & $630(40)$ & $210(40)$ \\
\hline $\mathrm{N}(1)$ & $60(40)$ & $360(50)$ & $40(40)$ & $160(30)$ & $-10(30)$ & $140(30)$ \\
\hline $\mathrm{N}(3)$ & $240(50)$ & $220(50)$ & $250(50)$ & $130(40)$ & $140(40)$ & $100(40)$ \\
\hline $\mathrm{N}(2)$ & $200(50)$ & $250(50)$ & $330(50)$ & $140(40)$ & $70(40)$ & $0(40)$ \\
\hline $\mathrm{N}(5)$ & $110(40)$ & $370(50)$ & $280(50)$ & $260(40)$ & $50(40)$ & $80(40)$ \\
\hline$C(45)$ & $530(70)$ & $550(80)$ & $310(60)$ & $410(60)$ & $350(60)$ & $480(60)$ \\
\hline $\mathrm{N}(4)$ & $160(40)$ & $510(60)$ & $280(50)$ & $190(50)$ & $30(40)$ & $100(40)$ \\
\hline $\mathrm{C}(18)$ & $410(70)$ & $460(70)$ & $460(70)$ & $320(60)$ & $180(60)$ & $160(60)$ \\
\hline C(36) & $140(50)$ & $770(90)$ & $40(50)$ & $170(50)$ & $50(40)$ & $250(50)$ \\
\hline C(31) & $280(60)$ & $380(70)$ & $270(60)$ & $240(50)$ & $-110(50)$ & $50(50)$ \\
\hline $\mathrm{C}(14)$ & $420(70)$ & $370(70)$ & $340(60)$ & $230(50)$ & $90(50)$ & $180(50)$ \\
\hline $\mathrm{C}(7)$ & $170(50)$ & $270(60)$ & $310(60)$ & $120(50)$ & $190(50)$ & $100(40)$ \\
\hline$C(15)$ & $270(60)$ & $440(70)$ & $510(70)$ & $290(60)$ & $260(50)$ & $90(50)$ \\
\hline $\mathrm{O}(1)$ & $750(70)$ & $660(70)$ & $1120(80)$ & $520(60)$ & $180(60)$ & $-40(50)$ \\
\hline$C(12)$ & $320(60)$ & $450(80)$ & $190(60)$ & $140(60)$ & $120(50)$ & $20(60)$ \\
\hline$C(40)$ & $250(60)$ & $520(80)$ & $590(80)$ & $450(70)$ & $-50(60)$ & $-120(60)$ \\
\hline $\mathrm{C}(5)$ & $420(70)$ & $360(70)$ & $430(70)$ & $150(60)$ & $250(60)$ & $150(60)$ \\
\hline$C(6)$ & $360(60)$ & $170(60)$ & $390(60)$ & $140(50)$ & $240(50)$ & $150(50)$ \\
\hline C(19) & $410(60)$ & $210(60)$ & $280(60)$ & $150(50)$ & $130(50)$ & $90(50)$ \\
\hline $\mathrm{O}(2)$ & $450(60)$ & $1010(80)$ & $1460(90)$ & $290(70)$ & $230(60)$ & $140(50)$ \\
\hline C(3) & $450(70)$ & $590(80)$ & $490(80)$ & $240(70)$ & $330(60)$ & $50(60)$ \\
\hline C(30) & $470(70)$ & $530(80)$ & $0(50)$ & $80(50)$ & $110(50)$ & $100(60)$ \\
\hline $\mathrm{C}(9)$ & $330(60)$ & $210(60)$ & $200(60)$ & $70(50)$ & $80(50)$ & $40(50)$ \\
\hline $\mathrm{C}(8)$ & $280(60)$ & $330(70)$ & $400(70)$ & $110(50)$ & $290(50)$ & $130(50)$ \\
\hline C(32) & $280(60)$ & $550(80)$ & $230(60)$ & $70(60)$ & $160(50)$ & $280(60)$ \\
\hline $\mathrm{C}(17)$ & $410(70)$ & $720(100)$ & $570(80)$ & $340(70)$ & $360(70)$ & $120(70)$ \\
\hline $\mathrm{C}(1)$ & $1130(120)$ & $290(70)$ & $750(100)$ & $210(70)$ & $480(90)$ & $70(70)$ \\
\hline$C(26)$ & $740(90)$ & $120(60)$ & $620(90)$ & $60(60)$ & $310(80)$ & $150(60)$ \\
\hline C(20) & $430(70)$ & $190(60)$ & $290(70)$ & $-70(50)$ & $230(60)$ & $-40(50)$ \\
\hline $\mathrm{C}(11)$ & $430(70)$ & $810(100)$ & $390(70)$ & $300(70)$ & $280(60)$ & $300(70)$ \\
\hline C(33) & $490(70)$ & $430(80)$ & $170(60)$ & $-70(50)$ & $50(50)$ & $300(60)$ \\
\hline$C(4)$ & $360(60)$ & $220(60)$ & $550(70)$ & $140(60)$ & $100(60)$ & $-60(50)$ \\
\hline C(29) & $400(70)$ & $650(80)$ & $330(70)$ & $370(60)$ & $280(60)$ & $310(60)$ \\
\hline C(28) & $440(70)$ & $640(90)$ & $440(80)$ & $310(70)$ & $100(60)$ & $330(70)$ \\
\hline$C(48)$ & $690(90)$ & $610(80)$ & $290(70)$ & $280(60)$ & $10(60)$ & $120(70)$ \\
\hline C(34) & $580(80)$ & $320(70)$ & $370(70)$ & $100(60)$ & $100(60)$ & $30(60)$ \\
\hline $\mathrm{C}(2)$ & $770(90)$ & $440(80)$ & $360(70)$ & $170(60)$ & $260(70)$ & $80(70)$ \\
\hline$C(16)$ & $370(70)$ & $570(80)$ & $860(100)$ & $470(80)$ & $420(70)$ & $170(60)$ \\
\hline$F(3)$ & 2180(130) & $950(70)$ & $1140(80)$ & $-100(70)$ & $850(80)$ & 250(80) \\
\hline C(13) & $500(70)$ & $370(70)$ & $260(60)$ & $90(50)$ & $-10(60)$ & $220(60)$ \\
\hline
\end{tabular}




\begin{tabular}{ccccccc}
$\mathrm{O}(3)$ & $860(70)$ & $1730(100)$ & $1030(80)$ & $840(80)$ & $280(60)$ & $800(70)$ \\
$\mathrm{C}(22)$ & $810(100)$ & $560(90)$ & $520(80)$ & $400(70)$ & $10(80)$ & $-210(70)$ \\
$\mathrm{C}(43)$ & $870(140)$ & $890(140)$ & $840(130)$ & $600(120)$ & $-220(110)$ & $-140(110)$ \\
$\mathrm{C}(41)$ & $240(70)$ & $1600(150)$ & $620(90)$ & $980(100)$ & $180(70)$ & $210(90)$ \\
$\mathrm{C}(39)$ & $590(80)$ & $940(100)$ & $280(70)$ & $220(70)$ & $210(60)$ & $420(70)$ \\
$\mathrm{C}(35)$ & $440(70)$ & $390(70)$ & $400(70)$ & $150(60)$ & $150(60)$ & $60(60)$ \\
$\mathrm{C}(10)$ & $310(70)$ & $460(70)$ & $570(80)$ & $160(60)$ & $60(60)$ & $-10(60)$ \\
$\mathrm{C}(21)$ & $650(90)$ & $660(100)$ & $540(90)$ & $340(80)$ & $-180(70)$ & $-110(80)$ \\
$\mathrm{C}(23)$ & $590(80)$ & $240(70)$ & $310(70)$ & $40(60)$ & $30(60)$ & $20(60)$ \\
$\mathrm{C}(25)$ & $940(110)$ & $380(80)$ & $470(80)$ & $210(70)$ & $230(80)$ & $160(80)$ \\
$\mathrm{C}(37)$ & $480(80)$ & $970(100)$ & $630(90)$ & $450(80)$ & $190(70)$ & $300(70)$ \\
$\mathrm{O}(5)$ & $1040(90)$ & $1400(100)$ & $1010(90)$ & $-530(80)$ & $490(70)$ & $-210(80)$ \\
$\mathrm{O}(6)$ & $1480(120)$ & $1970(140)$ & $2400(160)$ & $1010(120$ & $1300(120)$ & $500(100)$ \\
$\mathrm{C}(44)$ & $1210(140)$ & $930(120)$ & $650(100)$ & $570(90)$ & $220(100)$ & $620(110)$ \\
$\mathrm{F}(4)$ & $1630(110)$ & $1120(80)$ & $1940(120)$ & $-10(80)$ & $640(100)$ & $-130(80)$ \\
$\mathrm{C}(42)$ & $310(80)$ & $1340(150)$ & $1100(140)$ & $1160(130)$ & $-350(90)$ & $-400(100)$ \\
$\mathrm{C}(46)$ & $480(90)$ & $1770(170)$ & $1100(130)$ & $820(130)$ & $330(90)$ & $210(100)$ \\
$\mathrm{C}(27)$ & $670(90)$ & $820(110)$ & $580(90)$ & $440(80)$ & $80(80)$ & $390(80)$ \\
$\mathrm{C}(49)$ & $1030(160)$ & $2100(200)$ & $900(150)$ & $990(170)$ & $250(130)$ & $530(170)$ \\
$\mathrm{F}(1)$ & $1960(120)$ & $1450(90)$ & $1410(90)$ & $220(70)$ & $1210(100)$ & $-520(90)$ \\
$\mathrm{C}(38)$ & $680(100)$ & $1210(140)$ & $660(100)$ & $250(100)$ & $100(80)$ & $290(100)$ \\
$\mathrm{O}(4)$ & $1180(120)$ & $3200(200)$ & $3800(300)$ & $400(200)$ & $-700(150)$ & $1540(150)$ \\
$\mathrm{C}(24)$ & $650(100)$ & $480(90)$ & $700(100)$ & $130(80)$ & $30(80)$ & $50(70)$ \\
$\mathrm{F}(2)$ & $1990(130)$ & $2110(130)$ & $1220(90)$ & $1110(90)$ & $550(90)$ & $550(100)$ \\
$\mathrm{F}(6)$ & $2130(130)$ & $1670(110)$ & $1320(100)$ & $90(90)$ & $420(100)$ & $-170(100)$ \\
$\mathrm{C}(50)$ & $1300(180)$ & $1800(200)$ & $1440(180)$ & $-1320(180)$ & $1170(160)$ & $-1070(170)$ \\
$\mathrm{C}(47)$ & $1030(140)$ & $1170(150)$ & $1410(160)$ & $780(130)$ & $-370(120)$ & $-300(120)$ \\
$\mathrm{F}(5)$ & $6600(500)$ & $12500(900)$ & $8800(700)$ & $9400(800)$ & $5300(500)$ & $7700(600)$ \\
\hline
\end{tabular}




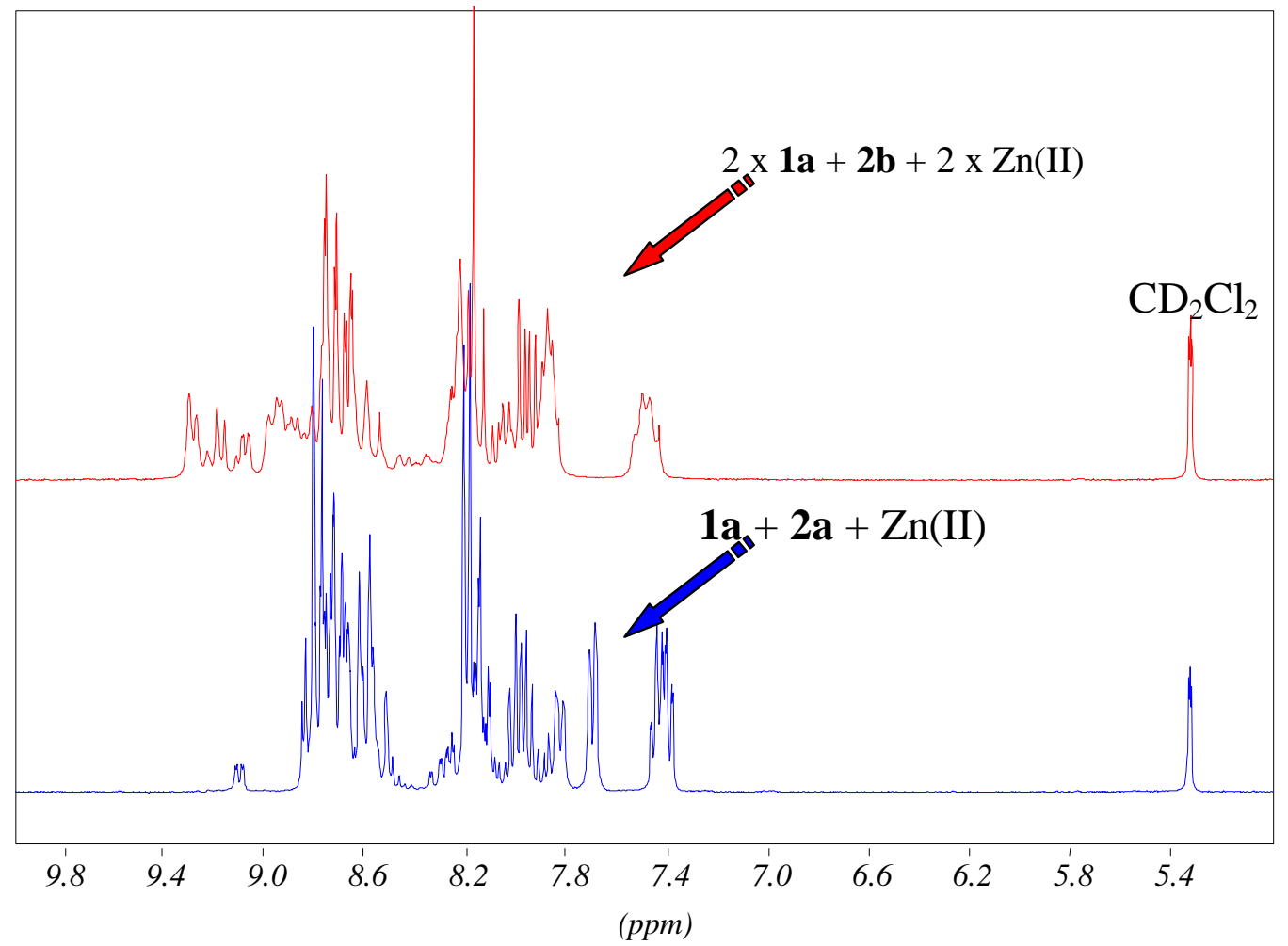

Figure S1. ${ }^{1} \mathrm{H}$ NMR spectrum of the aromatic region of two reaction mixtures. Top: 2 x 1a + $\mathbf{2 b}+2$ x Zn(II) and bottom: $\mathbf{1 a}+\mathbf{2 b}+\mathrm{Zn}(\mathrm{II})$. 


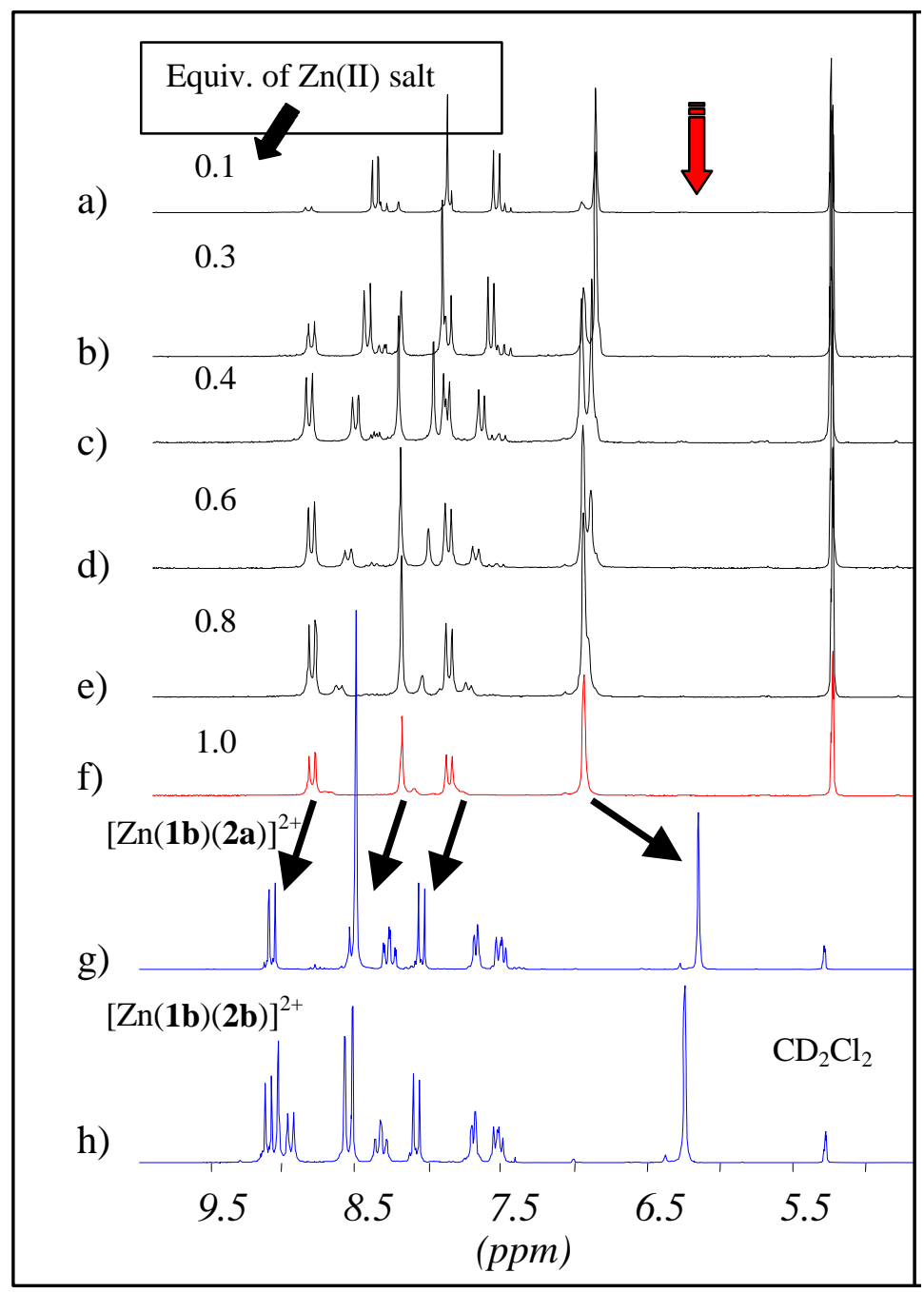

Figure S2. ${ }^{1} \mathrm{H}$ NMR of the aromatic region showing the changes in the signals of $\mathbf{1 b}$ upon $\mathrm{Zn}(\mathrm{II})$ coordination (0.1-1.0 equiv. of Zn(II): a)-f). Spectra g) and h) display a selected region of heteroleptic complexes $[\operatorname{Zn}(\mathbf{1 b})(\mathbf{2 a})]^{2+}$ and $\left[\operatorname{Zn}(\mathbf{1 b})_{2}(\mathbf{2 b})\right]^{4+}$. 


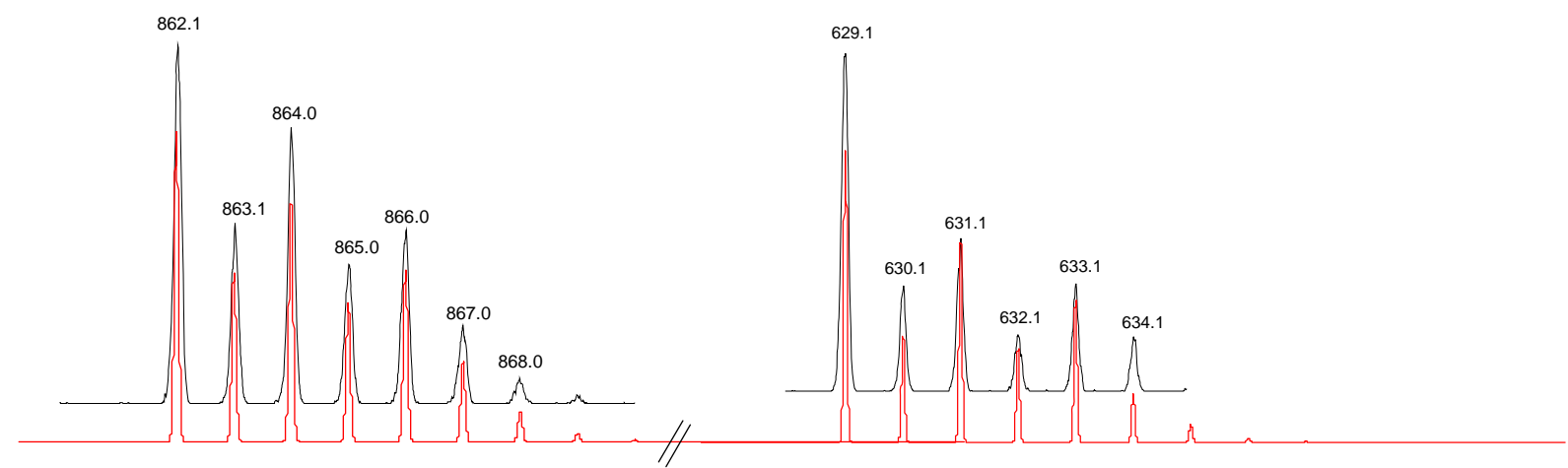

Figure S3. Isotopic distribution of the peaks at $\mathrm{m} / \mathrm{z}=862.2$ (red lines are calculated and black lines are experimental) of the reaction mixture obtained when $\mathbf{1} \mathbf{b}$ and $\mathbf{2 a}$ were reacted with $\left[\mathrm{Zn}(\mathrm{OTf})_{2}\right]$ in dichloromethane/methanol (8:2) at rt. Calcd. for $\left[\mathrm{ZnC}_{45} \mathrm{H}_{39} \mathrm{~N}_{5} * \mathrm{OTf}\right]^{+}\left[\mathrm{M}^{+}\right](\%)$ : $\mathrm{m} / \mathrm{z}$ 864.3. Found: $\mathrm{m} / \mathrm{z} 862.2$ (100).

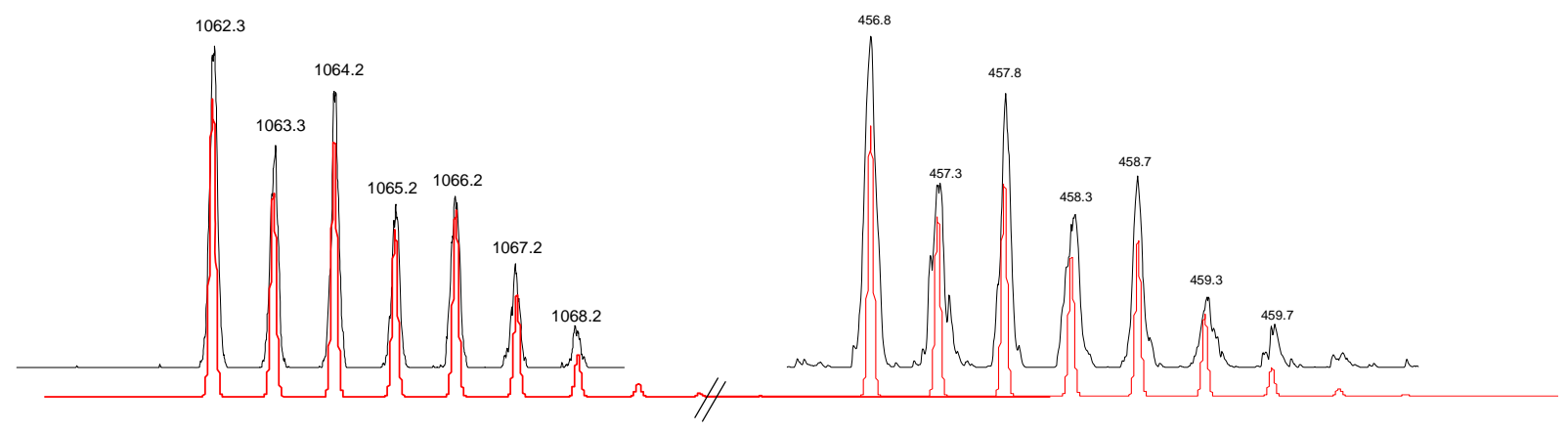

Figure S4. Isotopic distribution of the peaks at $\mathrm{m} / \mathrm{z}=1062.3$ and 457.2 (red lines are calculated and black lines are experimental) of the reaction mixture obtained when 1c and 2a were reacted with $\left[\mathrm{Zn}(\mathrm{OTf})_{2}\right]$ in dichloromethane/methanol (8:2) at rt. Calcd. for $\left[\mathrm{ZnC}_{57} \mathrm{H}_{63} \mathrm{~N}_{5} \mathrm{O}_{2} * \mathrm{OTf}\right]^{+}\left[\mathrm{M}^{+}\right]$(\%): $\mathrm{m} / \mathrm{z}$ 1064.6. Found: $\mathrm{m} / \mathrm{z} 1062.3$ (100). [ $\left.\mathrm{ZnC}_{57} \mathrm{H}_{63} \mathrm{~N}_{5} \mathrm{O}_{2}\right]^{2+}$ $\left[\mathrm{M}^{2+}\right]$ (\%): $\mathrm{m} / \mathrm{z}$ 457.8. Found: $\mathrm{m} / \mathrm{z} 457.3$ (38). 


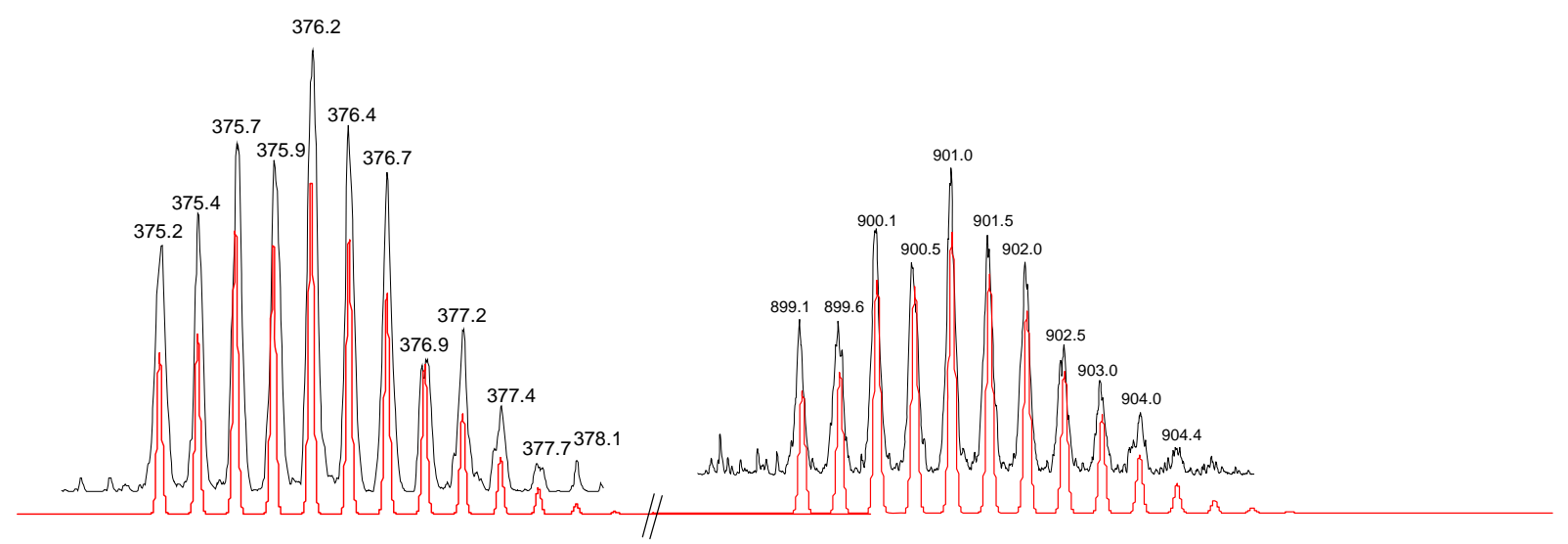

Figure S5. ESI-MS spectrum and isotopic distribution of the peaks at $\mathrm{m} / \mathrm{z}=900.4$ and 376.5 (red lines are calculated and black lines are experimental) of the reaction mixture obtained when $\mathbf{1 b}$ and $\mathbf{2 b}$ were reacted with $\left[\mathrm{Zn}(\mathrm{OTf})_{2}\right]$ in acetonitrile at rt. Calcd. for $\left[\mathrm{Zn}_{2} \mathrm{C}_{96} \mathrm{H}_{80} \mathrm{~N}_{10} * 2 \mathrm{OTf}\right]^{2+} \quad\left[\mathrm{M}^{2+}\right] \quad(\%): \quad m / z \quad 901.3$. Found: $\mathrm{m} / \mathrm{z} 900.4$ (11). Calcd. for $\left[\mathrm{Zn}_{2} \mathrm{C}_{96} \mathrm{H}_{80} \mathrm{~N}_{10}\right]^{4+}\left[\mathrm{M}^{4+}\right]$ (\%): $\mathrm{m} / \mathrm{z}$ 376.1. Found: $\mathrm{m} / \mathrm{z} 376.5$ (100).

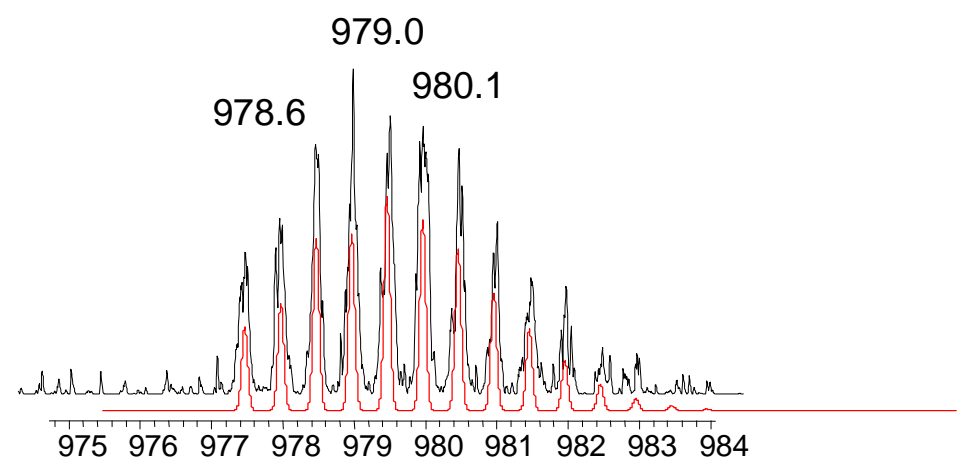

Figure S6. ESI-MS spectrum and isotopic distribution of the peak at $\mathrm{m} / \mathrm{z}=979.0$ (red lines are calculated and black lines are experimental) of the reaction mixture obtained when $\mathbf{1 b}$ and 2c were reacted with $\left[\mathrm{Zn}(\mathrm{OTf})_{2}\right]$ in dichloromethane/methanol (8:2) at rt. Calcd. for $\left[\mathrm{Zn}_{2} \mathrm{C}_{108} \mathrm{H}_{92} \mathrm{~N}_{10} * 2 \mathrm{OTf}\right]^{2+}\left[\mathrm{M}^{2+}\right]$ (\%): $\mathrm{m} / \mathrm{z}$ 979.4. Found: $\mathrm{m} / \mathrm{z} 979.0$ (100). Calcd. for $\left[\mathrm{Zn}_{2} \mathrm{C}_{108} \mathrm{H}_{92} \mathrm{~N}_{10} * \mathrm{OTf}\right]^{3+}\left[\mathrm{M}^{3+}\right](\%): \mathrm{m} / \mathrm{z}$ 603.3. Found: $\mathrm{m} / \mathrm{z} 604.1$ (74). 

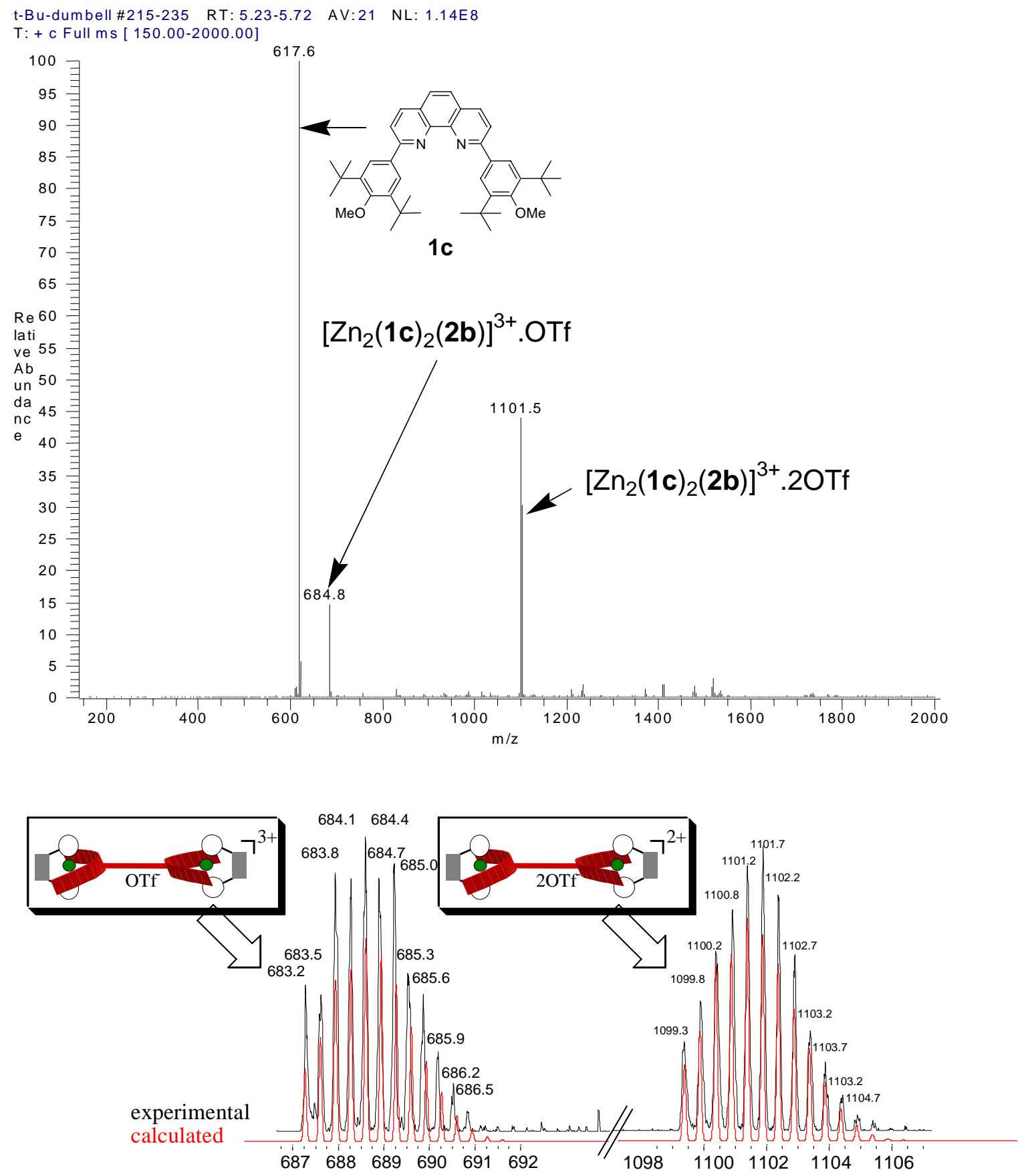

Figure S7. ESI-MS spectrum and isotopic distribution of the peaks at $\mathrm{m} / \mathrm{z}=1101.5$ and 684.8 (red lines are calculated and black lines are experimental) of the reaction mixture obtained when 1c and 2b were reacted with $\left[\mathrm{Zn}(\mathrm{OTf})_{2}\right.$ ] in acetonitrile at rt. Calcd. for [ $\mathrm{Zn}_{2}$ $\left.\mathrm{C}_{120} \mathrm{H}_{128} \mathrm{~N}_{10} \mathrm{O}_{4} * 2 \mathrm{OTf}\right]^{2+}\left[\mathrm{M}^{2+}\right]$ (\%): $\mathrm{m} / \mathrm{z}$ 1101.6. Found: $\mathrm{m} / \mathrm{z} 1101.5$ (44). Calcd. for $\mathrm{Zn}_{2}$ $\left.\mathrm{C}_{120} \mathrm{H}_{128} \mathrm{~N}_{10} \mathrm{O}_{4} * \mathrm{OTf}\right]^{3+}\left[\mathrm{M}^{3+}\right.$ ] (\%): $\mathrm{m} / \mathrm{z}$ 684.7. Found: $\mathrm{m} / \mathrm{z} 684.8$ (16). 


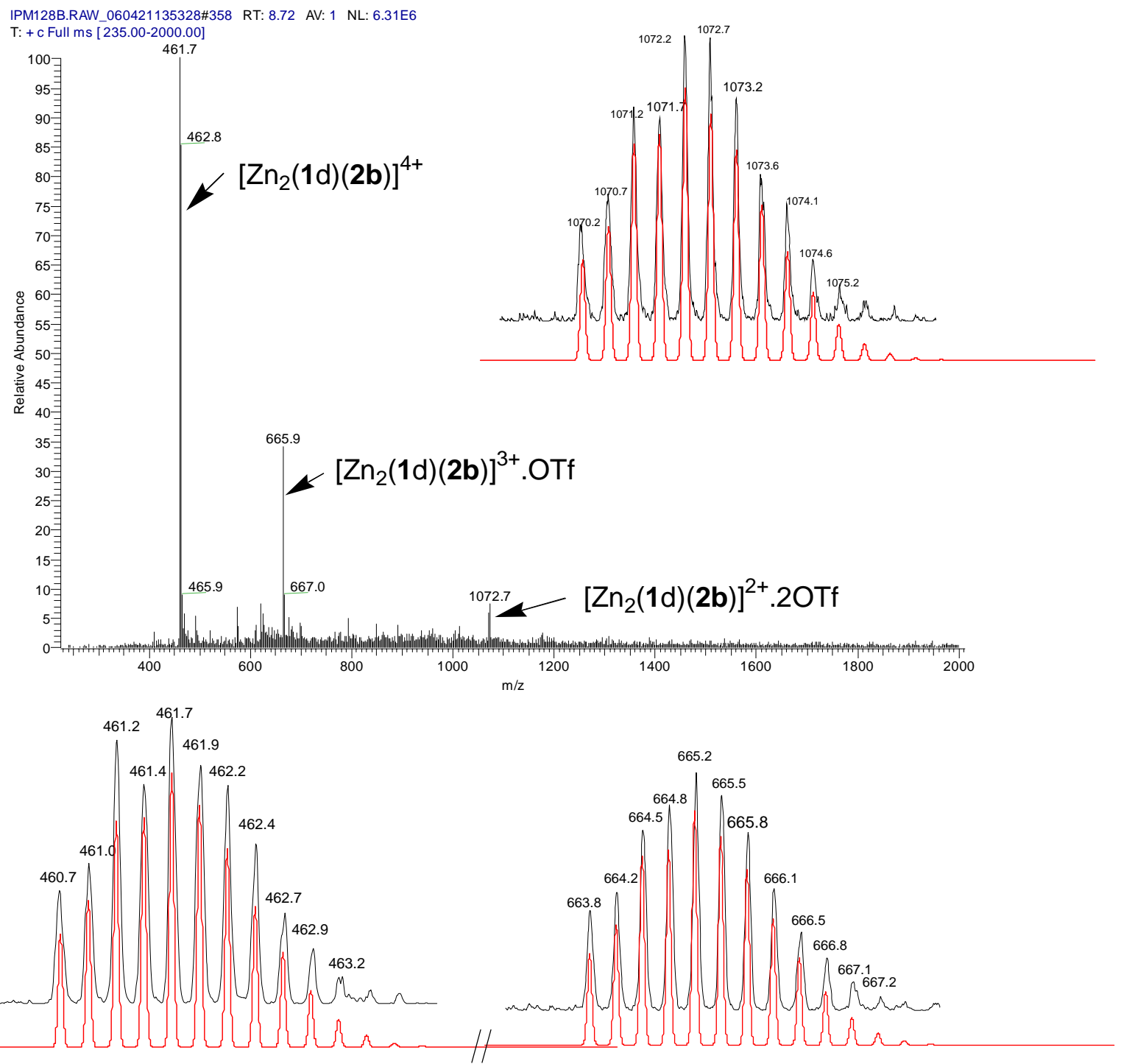

Figure S8. ESI-MS spectrum and isotopic distribution of the peaks at $\mathrm{m} / \mathrm{z}=1101.5$ and 684.8 (red lines are calculated and black lines are experimental) of the reaction mixture obtained when $\mathbf{1 d}$ and $\mathbf{2 b}$ were reacted with $\left[\mathrm{Zn}(\mathrm{OTf})_{2}\right]$ in acetonitrile at rt. Calcd. for [ $\mathrm{Zn}_{2}$ $\left.\mathrm{C}_{112} \mathrm{H}_{102} \mathrm{~N}_{10} \mathrm{O}_{8} * 2 \mathrm{OTf}\right]^{2+}\left[\mathrm{M}^{2+}\right]$ (\%): $\mathrm{m} / \mathrm{z}$ 1072.5. Found: $\mathrm{m} / \mathrm{z} 1072.7$ (7). Calcd. for $\mathrm{Zn}_{2}$ $\left.\mathrm{C}_{120} \mathrm{H}_{128} \mathrm{~N}_{10} \mathrm{O}_{4} * \mathrm{OTf}\right]^{3+}\left[\mathrm{M}^{3+}\right] \quad(\%): \mathrm{m} / \mathrm{z}$ 665.3. Found: $\mathrm{m} / \mathrm{z} 665.9$ (36). Calcd. for $\mathrm{Zn}_{2}$ $\left.\mathrm{C}_{120} \mathrm{H}_{128} \mathrm{~N}_{10} \mathrm{O}_{4}\right]^{4+}\left[\mathrm{M}^{4+}\right]$ (\%): $\mathrm{m} / \mathrm{z}$ 461.7. Found: $\mathrm{m} / \mathrm{z} 461.7$ (100). 

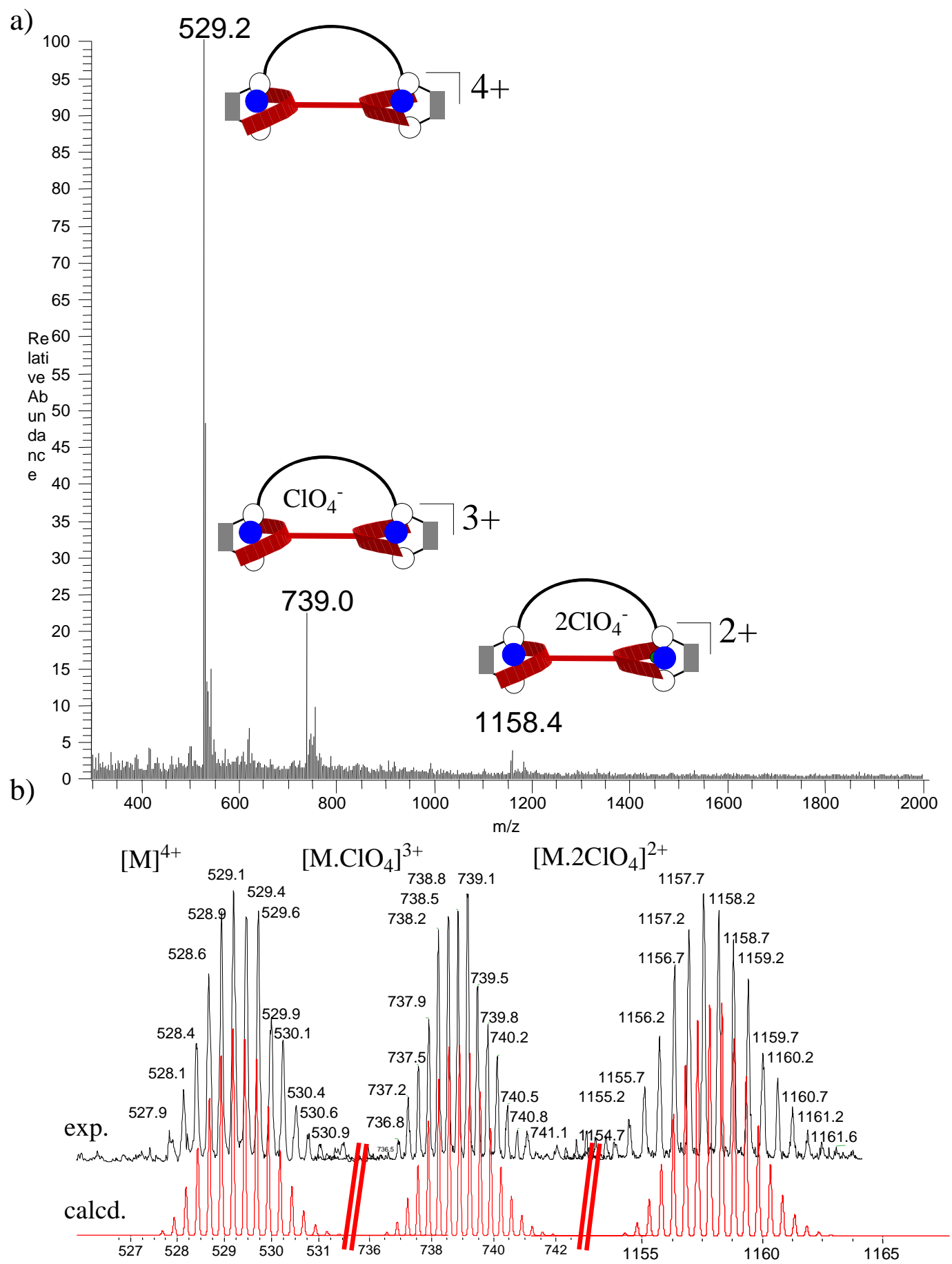

Figure S9. ESI-MS spectrum and isotopic distributions of some selected peaks (red lines are calculated and black lines are experimental) of the reaction mixture obtained when $\left[\mathrm{Zn}_{2}(\mathbf{1 d})(\mathbf{2 b})\right]^{4+}$ was reacted with $\left[\mathrm{Hg}\left(\mathrm{ClO}_{4}\right)_{2}\right]$ in acetonitrile at rt. ESI-MS: Calcd. for $\left[\mathrm{Hg}_{2} \mathrm{C}_{112} \mathrm{H}_{102} \mathrm{~N}_{10} \mathrm{O}_{8} * 2 \mathrm{ClO}_{4}\right]^{2+}\left[\mathrm{M}^{2+}\right](\%): \mathrm{m} / \mathrm{z}$ 1158.1. Found: $\mathrm{m} / \mathrm{z} 1158.4$ (5). Calcd. for $\left[\mathrm{Hg}_{2} \mathrm{C}_{112} \mathrm{H}_{102} \mathrm{~N}_{10} \mathrm{O}_{8} * \mathrm{ClO}_{4}\right]^{3+}\left[\mathrm{M}^{3+}\right]$ (\%): $\mathrm{m} / \mathrm{z}$ 738.9. Found: $\mathrm{m} / \mathrm{z} 739.0$ (24). Calcd. for $\left[\mathrm{Hg}_{2} \mathrm{C}_{112} \mathrm{H}_{102} \mathrm{~N}_{10} \mathrm{O}_{8}\right]^{4+}\left[\mathrm{M}^{4+}\right](\%): \mathrm{m} / z$ 529.3. Found: $\mathrm{m} / \mathrm{z} 529.2$ (100). 

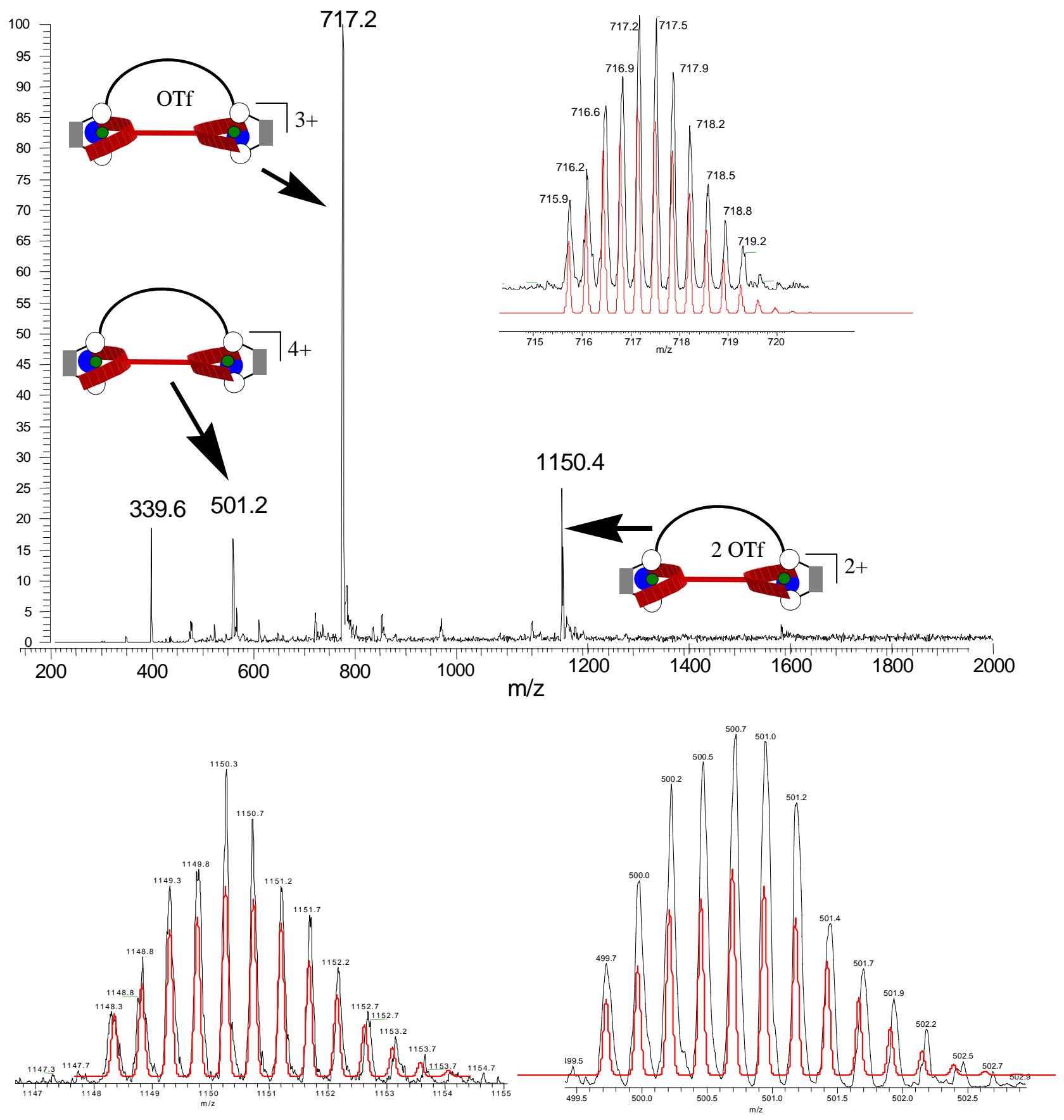

Figure S10. ESI-MS spectrum of the clip $\left[\mathrm{Zn}_{2}(\mathbf{1 d})(2 \mathrm{c})\right]^{4+}$ in $\mathrm{DCM}-\mathrm{CH}_{3} \mathrm{OH}(8: 2)$ at $\mathrm{rt}$ and isotopic distributions of some selected peaks (red lines are calculated and black lines are experimental): Calcd. for $\left[\mathrm{Zn}_{2} \mathrm{C}_{124} \mathrm{H}_{114} \mathrm{~N}_{10} \mathrm{O}_{8} * 2 \mathrm{OTf}\right]^{2+}\left[\mathrm{M}^{2+}\right]$ (\%): $\mathrm{m} / \mathrm{z}$ 1150.6. Found: $\mathrm{m} / \mathrm{z}$ 1150.7 (27). Calcd. for $\left[\mathrm{Zn}_{2} \mathrm{C}_{124} \mathrm{H}_{114} \mathrm{~N}_{10} \mathrm{O}_{8} * \mathrm{OTf}\right]^{3+}\left[\mathrm{M}^{3+}\right]$ (\%): $\mathrm{m} / \mathrm{z}$ 717.4. Found: $\mathrm{m} / \mathrm{z} 717.7$ (100). Calcd. for $\left[\mathrm{Zn}_{2} \mathrm{C}_{112} \mathrm{H}_{102} \mathrm{~N}_{10} \mathrm{O}_{8}\right]^{4+}\left[\mathrm{M}^{4+}\right]$ (\%): $\mathrm{m} / \mathrm{z}$ 500.7. Found: $\mathrm{m} / \mathrm{z} 501.2$ (19). 


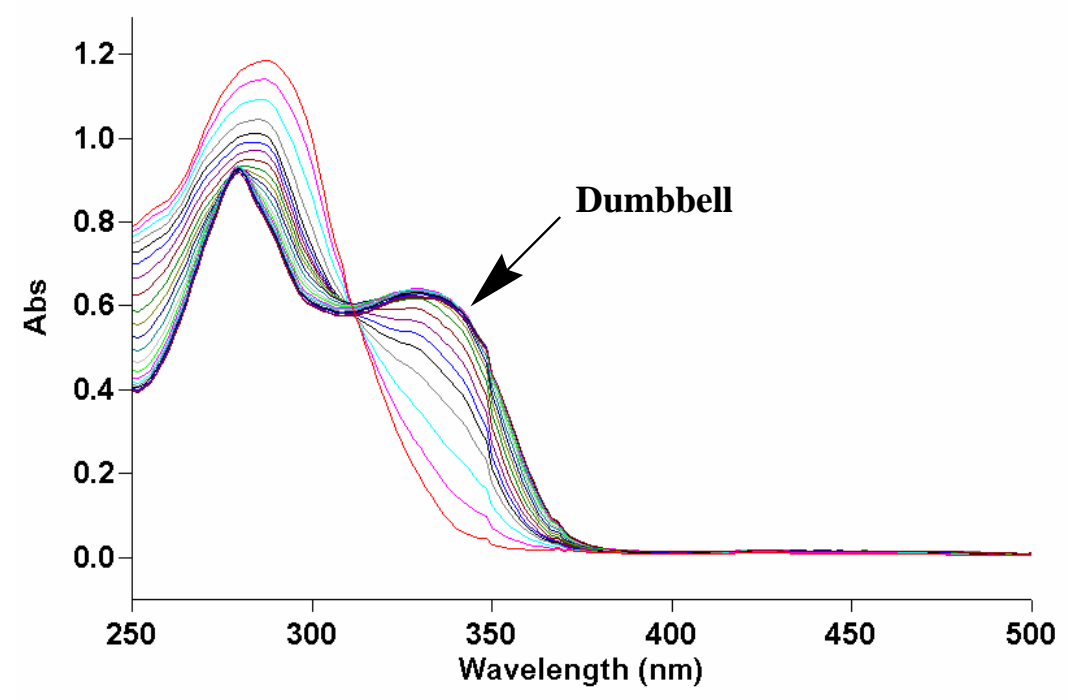

Figure S11. Spectrophotometric titration of the formation of dumbbell $\left[\mathrm{Zn}_{2}(\mathbf{1 b})_{2}(\mathbf{2 b})\right]^{4+}$ in dichloromethane/methanol (8:2). For the titration, a $\mathrm{Zn}(\mathrm{OTf})_{2}$ solution $\left(2.04 \times 10^{-4} \mathrm{M}\right)$ was added in $100 \mu \mathrm{L}$ increments to $2.0 \mathrm{~mL}$ of a $1.02 \times 10^{-5} \mathrm{M}$ solution of phenanthroline $\mathbf{1 b}$ and terpyridine $\mathbf{2 b}$. The absorption spectra were recorded at $25.0^{\circ} \mathrm{C}$.

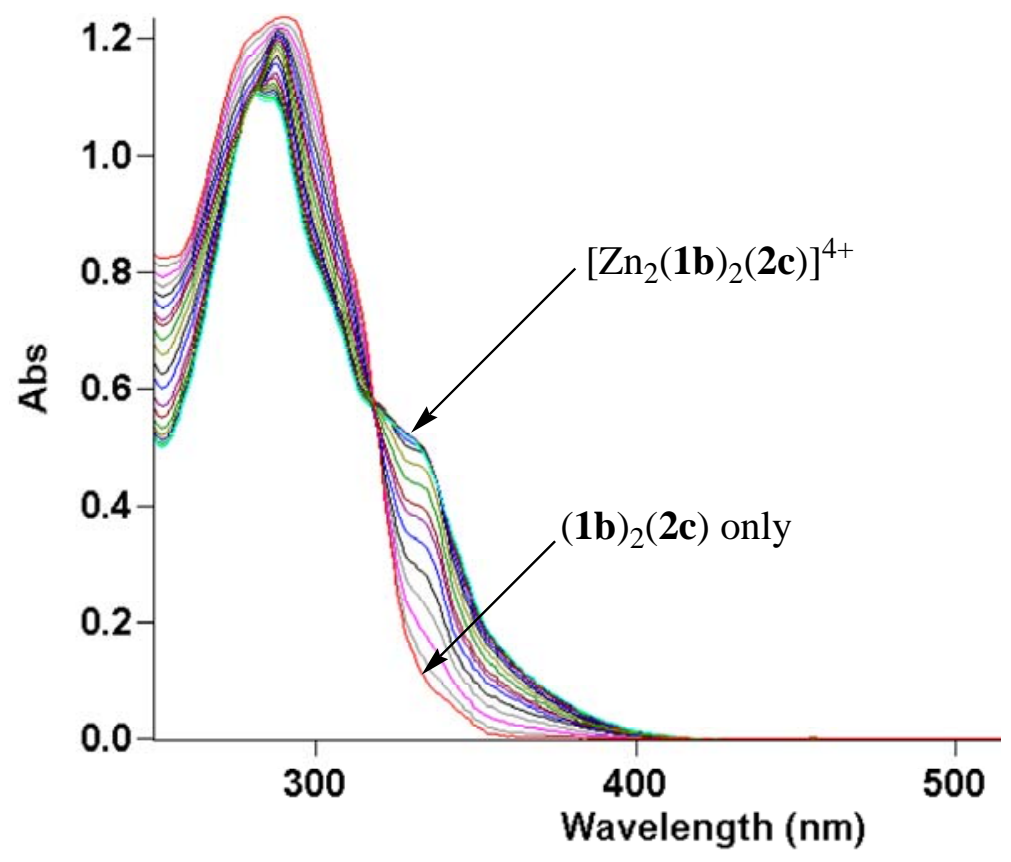

Figure S12. Spectrophotometric titration of the formation of dumbbell $\left[\mathrm{Zn}_{2}(\mathbf{1 b})_{2}(\mathbf{2 c})\right]^{4+}$ in dichloromethane/methanol (8:2). For the titration, a Zn(OTf $)_{2}$ solution $\left(2.04 \times 10^{-4} \mathrm{M}\right)$ was was added in $100 \mu \mathrm{L}$ increments to $2.0 \mathrm{~mL}$ of a $1.02 \times 10^{-5} \mathrm{M}$ solution of phenanthroline $\mathbf{1 b}$ and terpyridine 2c. The absorption spectra were recorded at $25.0^{\circ} \mathrm{C}$. 


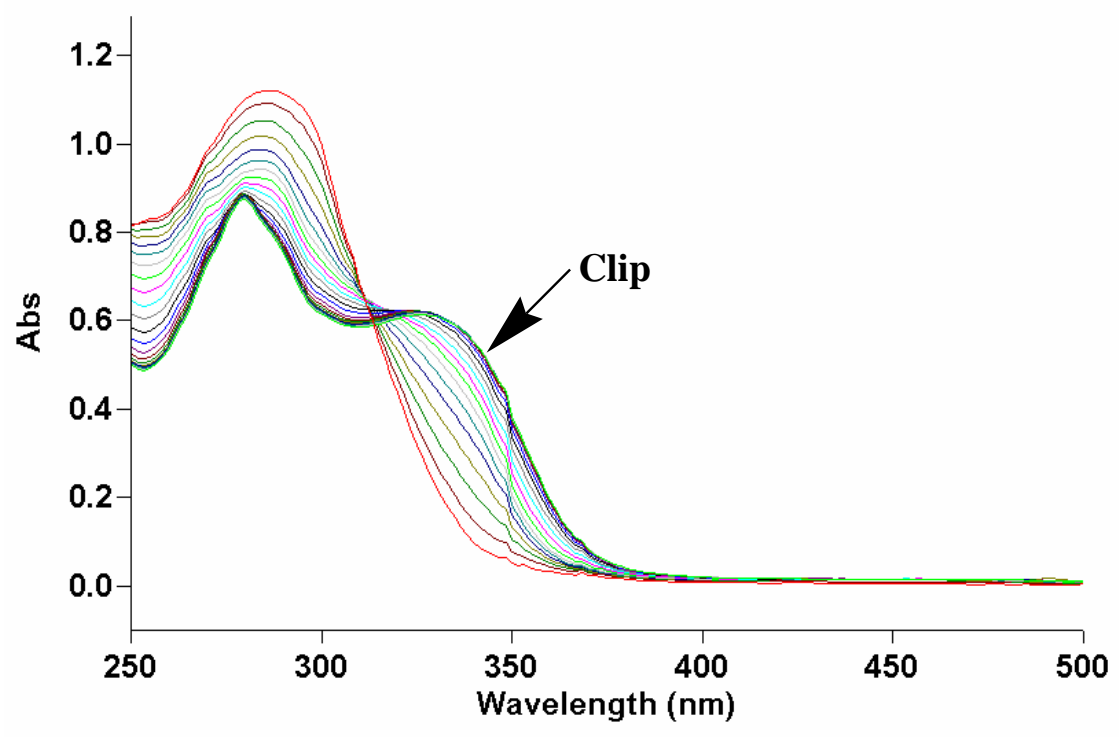

Figure S13. Spectrophotometric titration of the formation of clip $\left[\mathrm{Zn}_{2}(\mathbf{1 d})(\mathbf{2 b})\right]^{4+}$ in dichloromethane/methanol (8:2). For the titration, a $\mathrm{Zn}(\mathrm{OTf})_{2}$ solution $\left(2.04 \times 10^{-4} \mathrm{M}\right)$ was was added in $100 \mu \mathrm{L}$ increments to $2.0 \mathrm{~mL}$ of a $1.02 \times 10^{-5} \mathrm{M}$ solution of phenanthroline $1 \mathbf{d}$ and terpyridine $\mathbf{2 b}$. The absorption spectra were recorded at $25.0^{\circ} \mathrm{C}$.

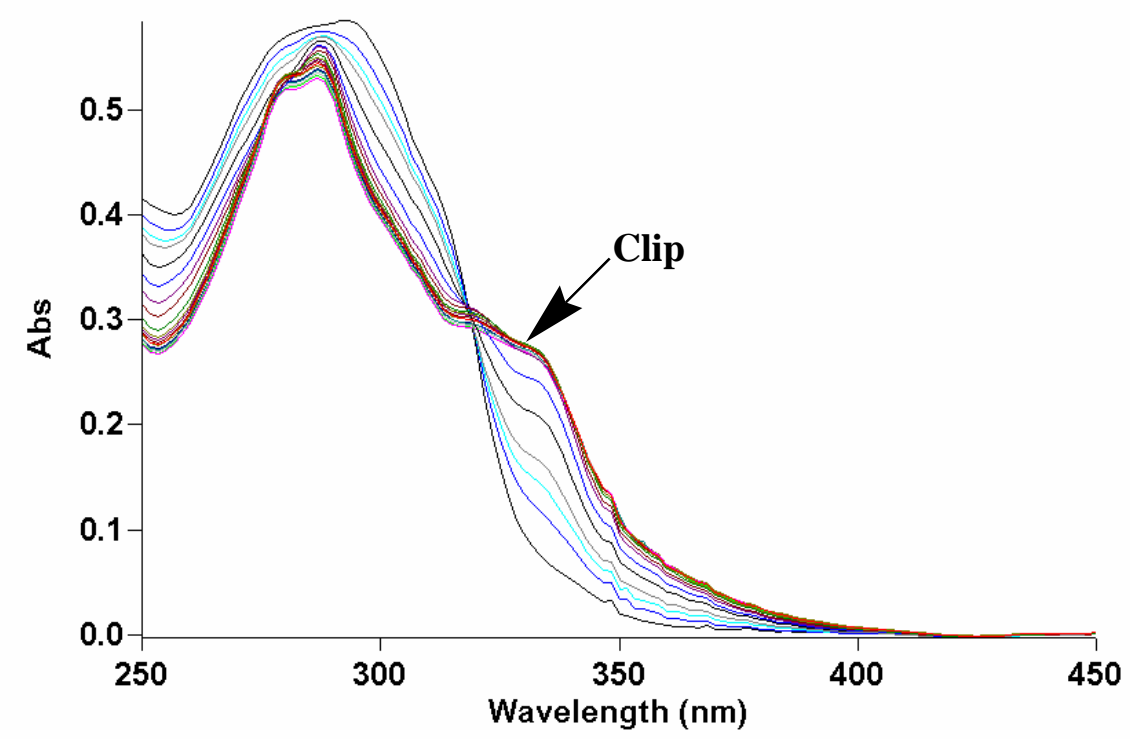

Figure S14. Spectrophotometric titration of the formation of clip $\left[\mathrm{Zn}_{2}(\mathbf{1 d})(\mathbf{2 c})\right]^{4+}$ in dichloromethane/methanol (8:2). For the titration, a $\mathrm{Zn}(\mathrm{OTf})_{2}$ solution $\left(2.04 \times 10^{-4} \mathrm{M}\right)$ was was added in $100 \mu \mathrm{L}$ increments to $2.0 \mathrm{~mL}$ of a $1.02 \times 10^{-5} \mathrm{M}$ solution of phenanthroline $1 \mathbf{d}$ and terpyridine 2c. The absorption spectra were recorded at $25.0^{\circ} \mathrm{C}$. 


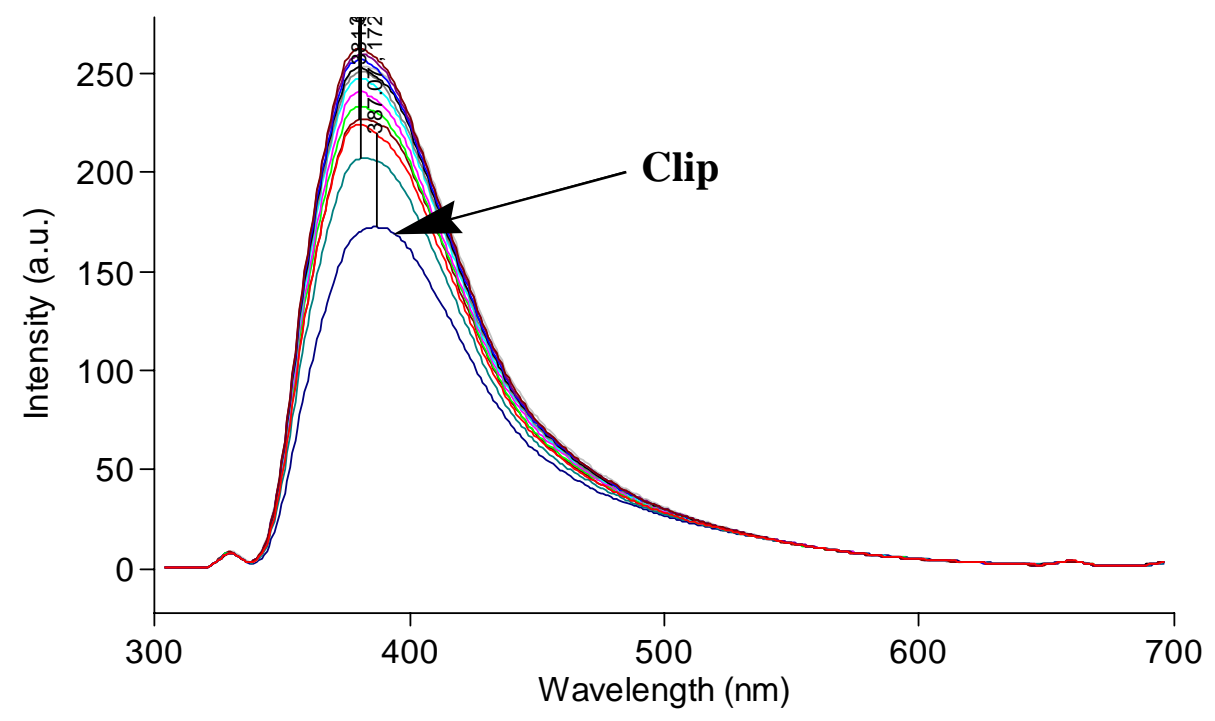

Figure S15. Change (enhancement) in the luminescence with time, after addition of $\mathrm{Hg}^{2+}(1: 1$ equivalent) to the clip $\left[\mathrm{Zn}_{2}(\mathbf{1 d})(\mathbf{2 b})\right]^{4+}$ in acetonitrile. The luminescence spectra were recorded at $25.0^{\circ} \mathrm{C}$ and excitation wavelength was $330 \mathrm{~nm}$.

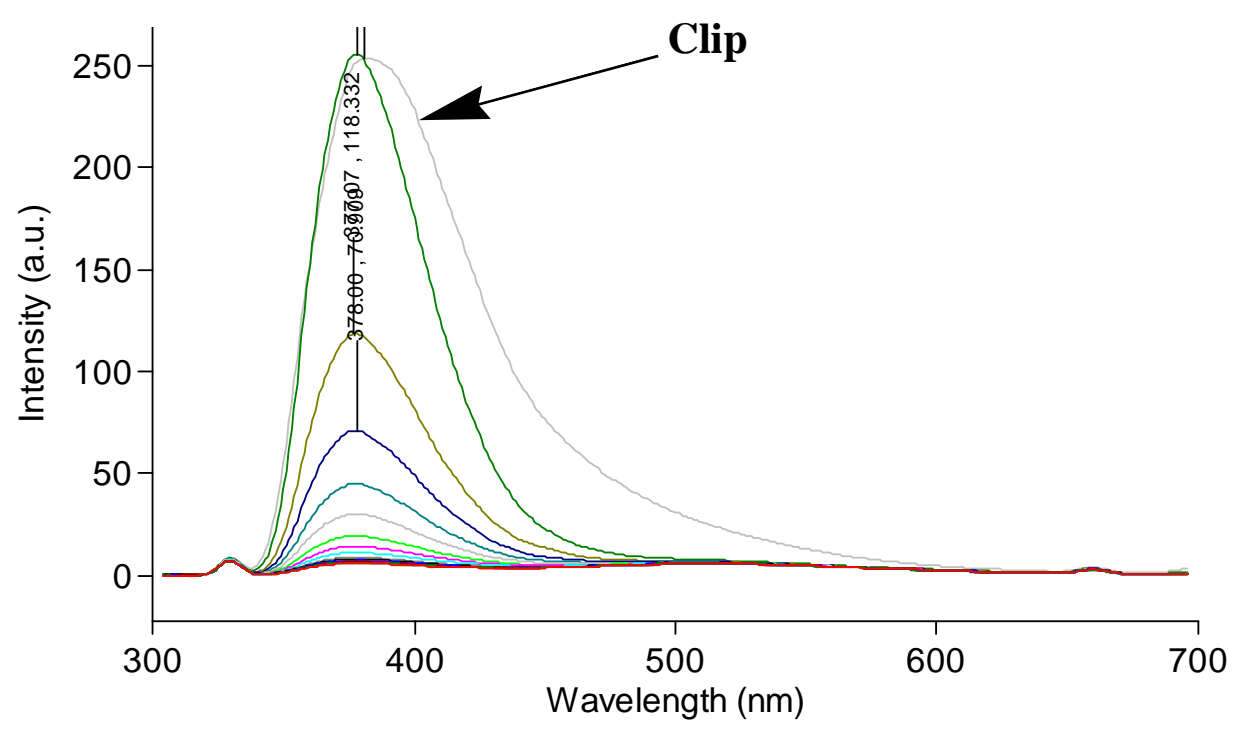

Figure S16. Quenching of luminescence with time (ca. $10 \mathrm{~min}$ for completion), after addition of $\mathrm{Hg}^{2+}$ (10:1 equivalent) to the clip $\left[\mathrm{Zn}_{2}(\mathbf{1 d})(\mathbf{2 b})\right]^{4+}$ in acetonitrile. The luminescence spectra were recorded at $25.0^{\circ} \mathrm{C}$ and excitation wavelength was $330 \mathrm{~nm}$. 


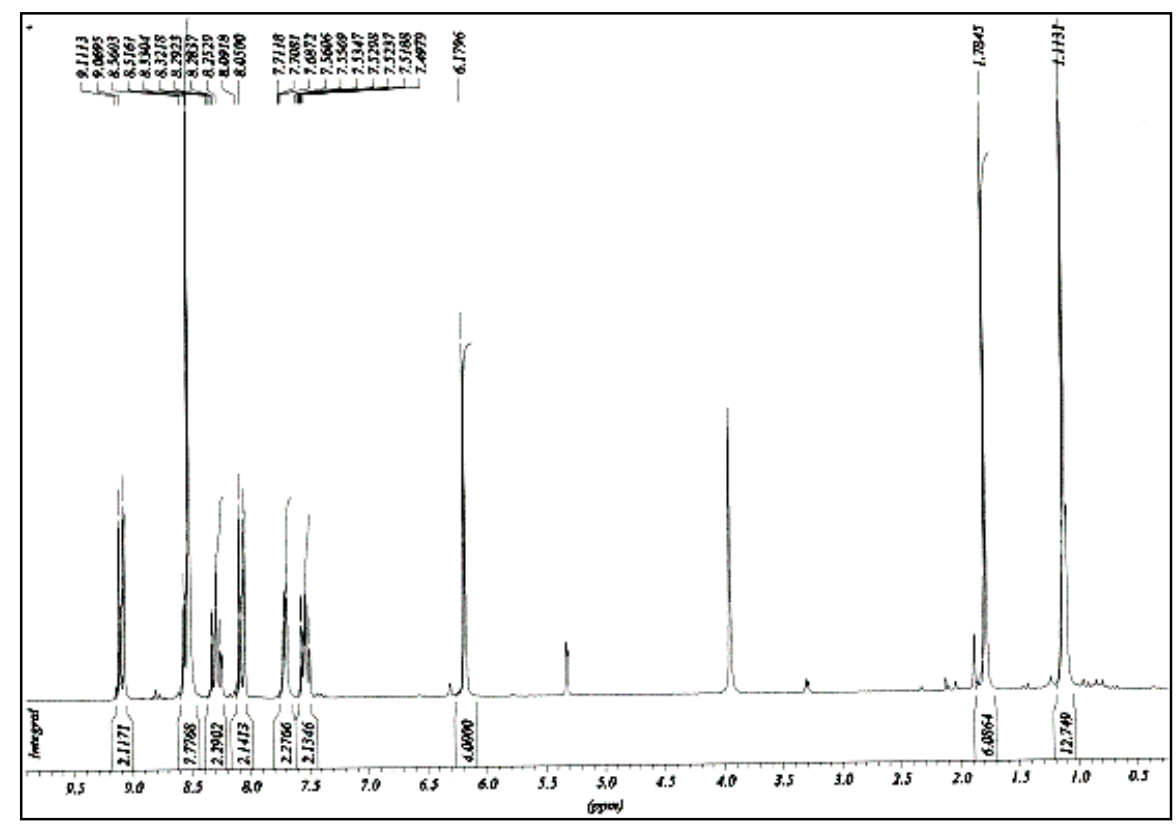

Figure S17. ${ }^{1} \mathrm{H} \mathrm{NMR}\left(\mathrm{CD}_{2} \mathrm{Cl}_{2} / \mathrm{CD}_{3} \mathrm{OD}(8: 2), 200 \mathrm{MHz}\right)$ spectrum of the complex $[\mathrm{Zn}(\mathbf{1 b})(\mathbf{2 a})](\mathrm{OTf})_{2}$.

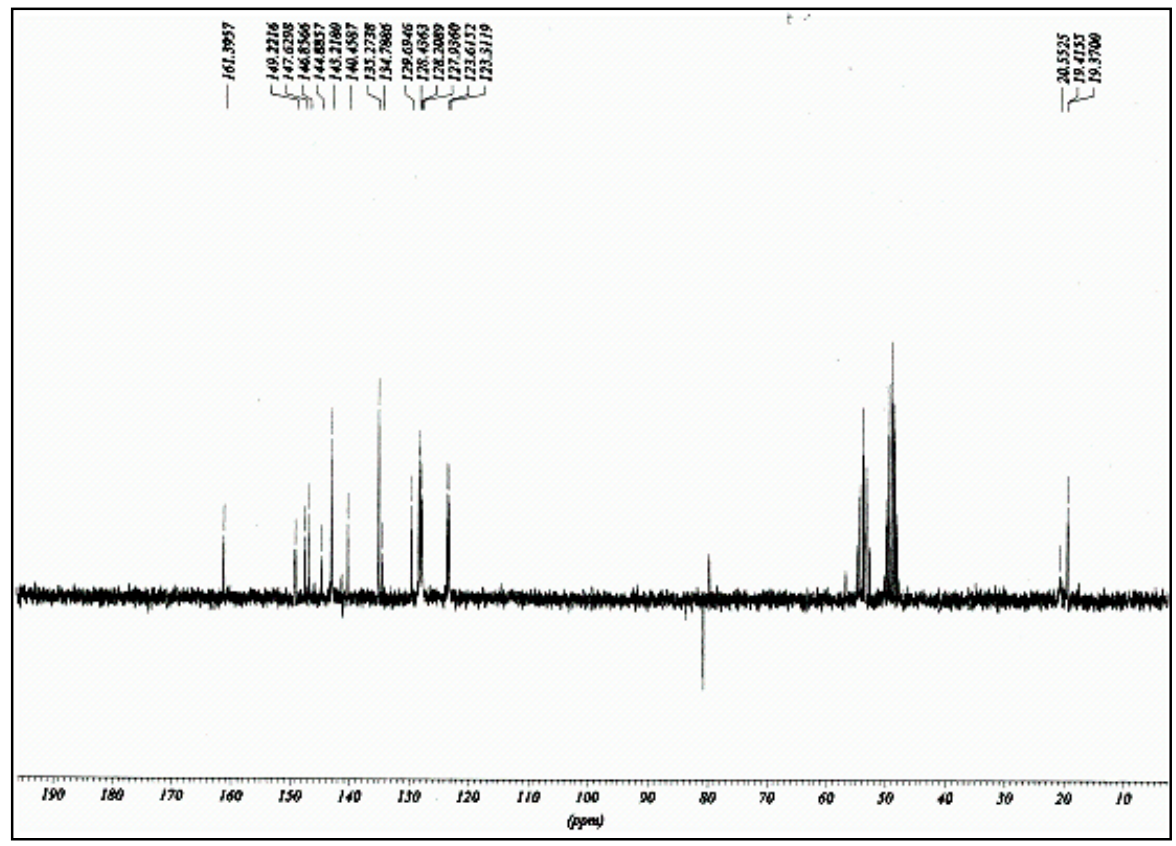

Figure S18. ${ }^{13} \mathrm{C}$ NMR $\left(\mathrm{CD}_{2} \mathrm{Cl}_{2} / \mathrm{CD}_{3} \mathrm{OD}(8: 2), 50 \mathrm{MHz}\right)$ spectrum of the complex [Zn(1b)(2a)] $(\mathrm{OTf})_{2}$. 


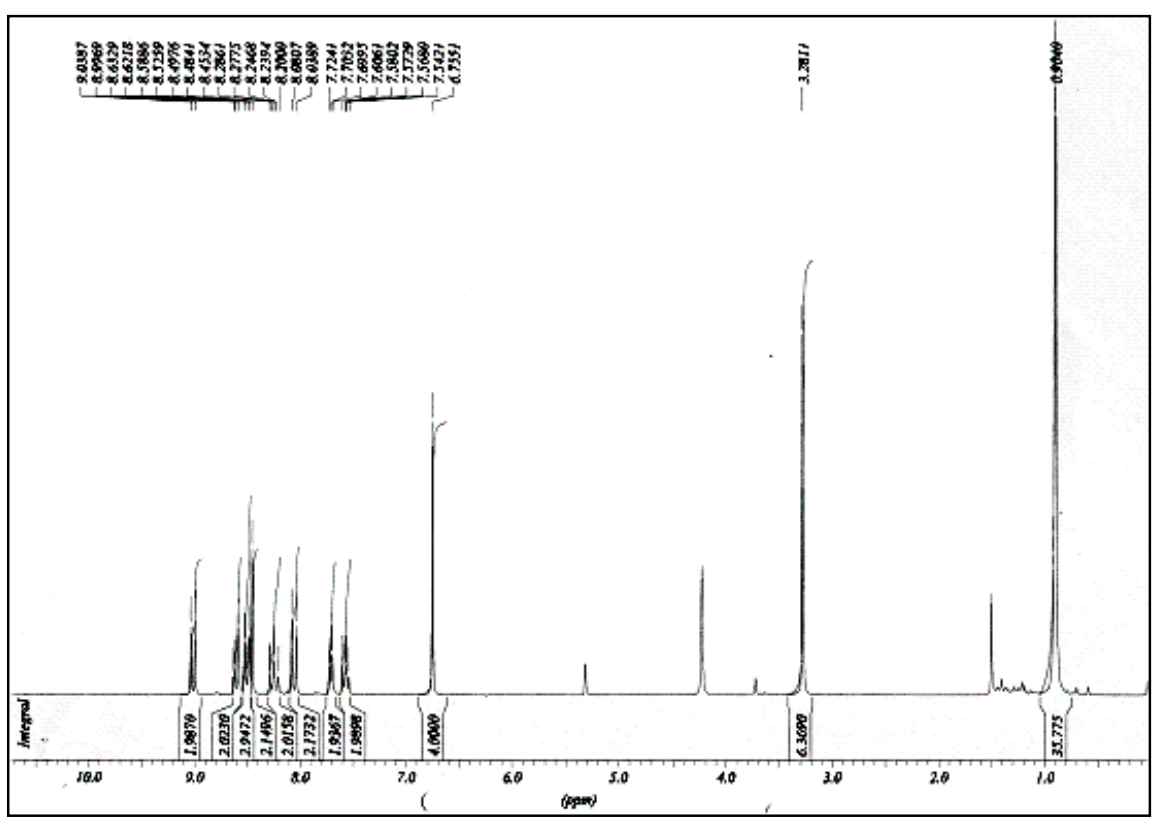

Figure S19. ${ }^{1} \mathrm{H}$ NMR $\left(\mathrm{CD}_{2} \mathrm{Cl}_{2} / \mathrm{CD}_{3} \mathrm{OD}(8: 2), 200 \mathrm{MHz}\right)$ spectrum of the complex $[\mathrm{Zn}(1 \mathbf{c})(\mathbf{2 a})](\mathrm{OTf})_{2}$.

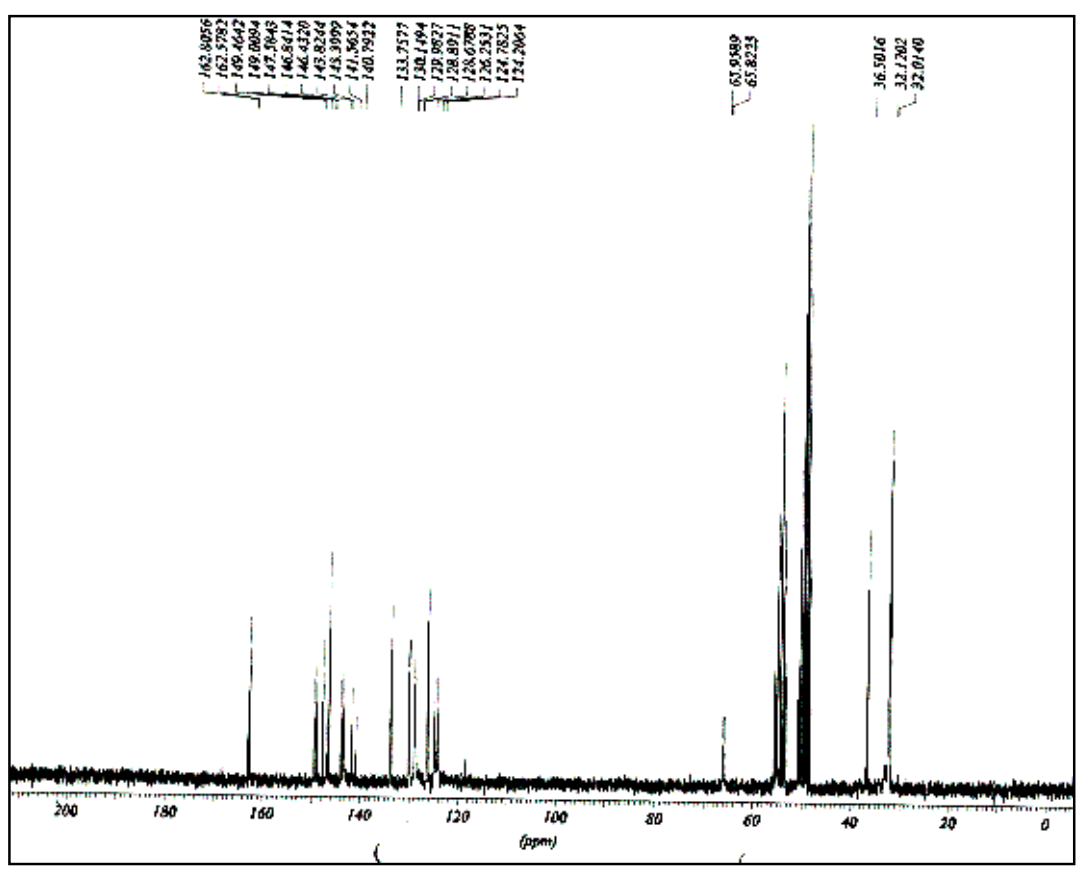

Figure S20. ${ }^{13} \mathrm{C}$ NMR $\left(\mathrm{CD}_{2} \mathrm{Cl}_{2} / \mathrm{CD}_{3} \mathrm{OD}(8: 2), 50 \mathrm{MHz}\right)$ spectrum of the complex [Zn(1c)(2a)] (OTf $)_{2}$. 


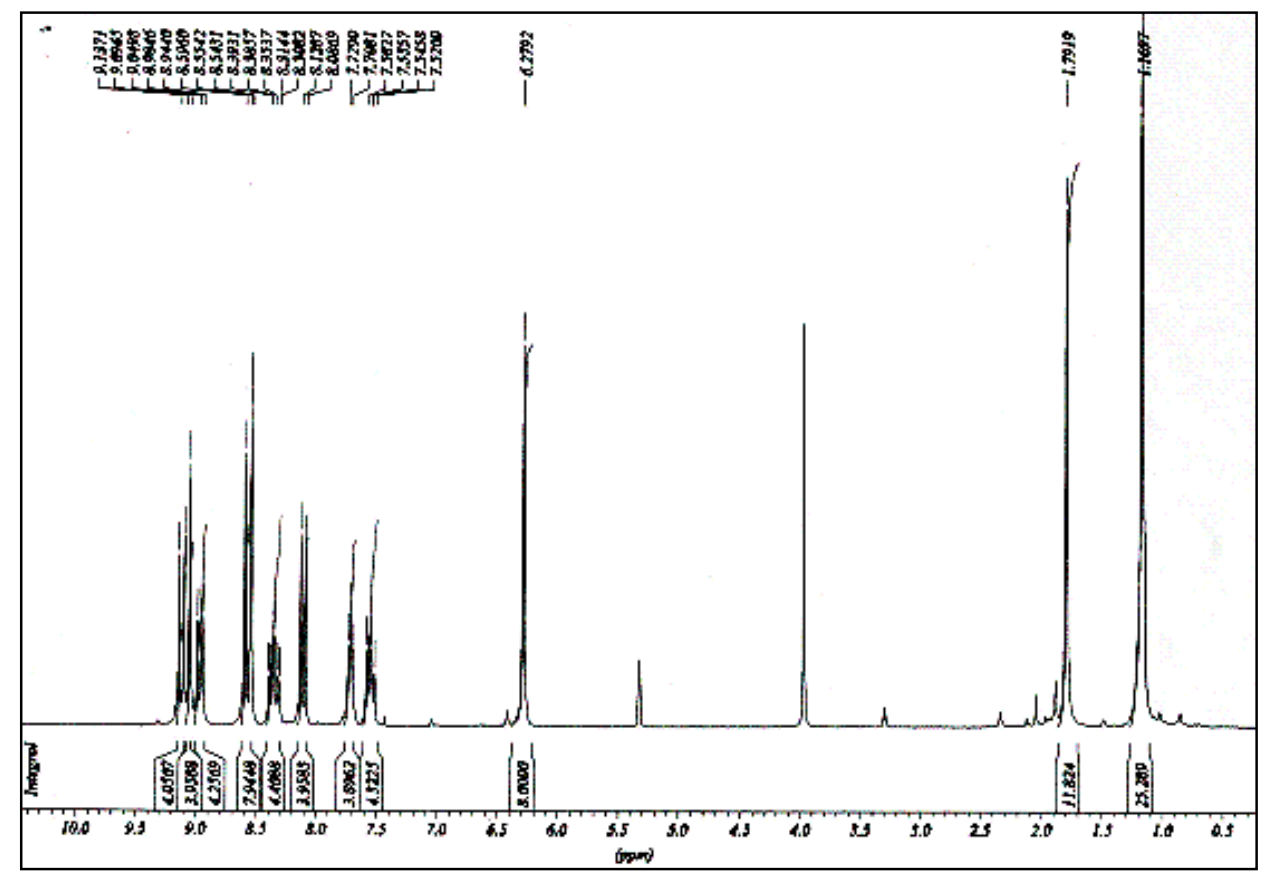

Figure S21. ${ }^{1} \mathrm{H}$ NMR $\left(\mathrm{CD}_{2} \mathrm{Cl}_{2} / \mathrm{CD}_{3} \mathrm{OD}(8: 2), 200 \mathrm{MHz}\right)$ spectrum of the complex $\left[\mathrm{Zn}_{2}(\mathbf{1 b})_{2}(\mathbf{2 b})\right](\mathrm{OTf})_{4}$.

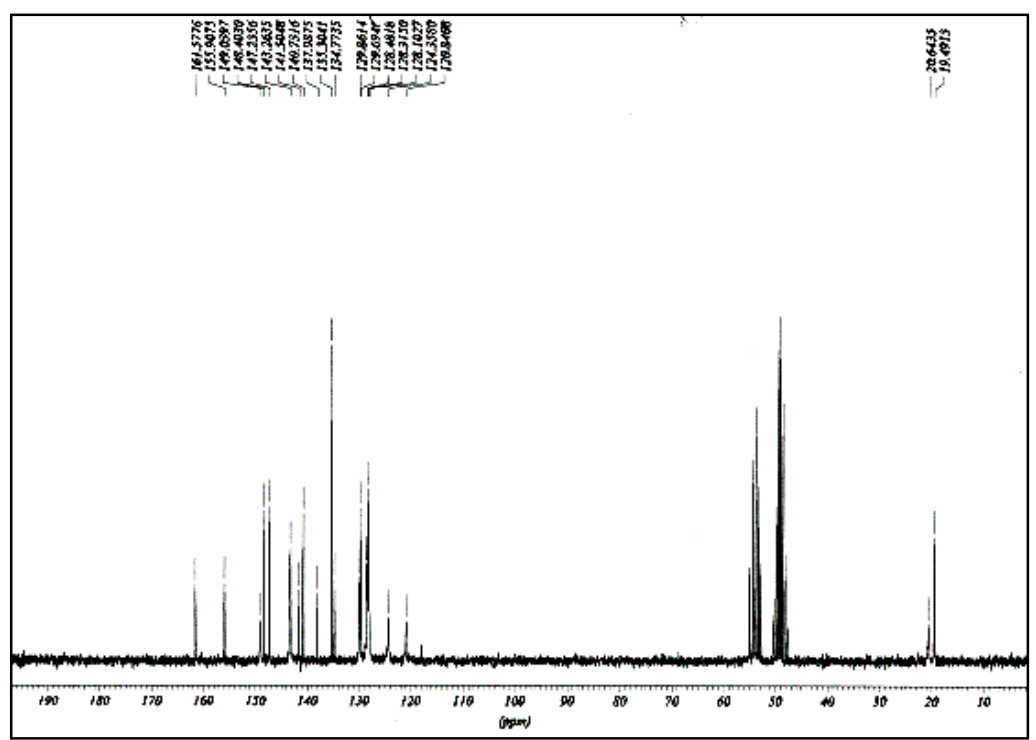

Figure S22. ${ }^{13} \mathrm{C}$ NMR $\left(\mathrm{CD}_{2} \mathrm{Cl}_{2} / \mathrm{CD}_{3} \mathrm{OD}(8: 2), 50 \mathrm{MHz}\right)$ spectrum of the complex $\left[\mathrm{Zn}_{2}(\mathbf{1 b})_{2}(\mathbf{2 b})\right](\mathrm{OTf})_{4}$. 


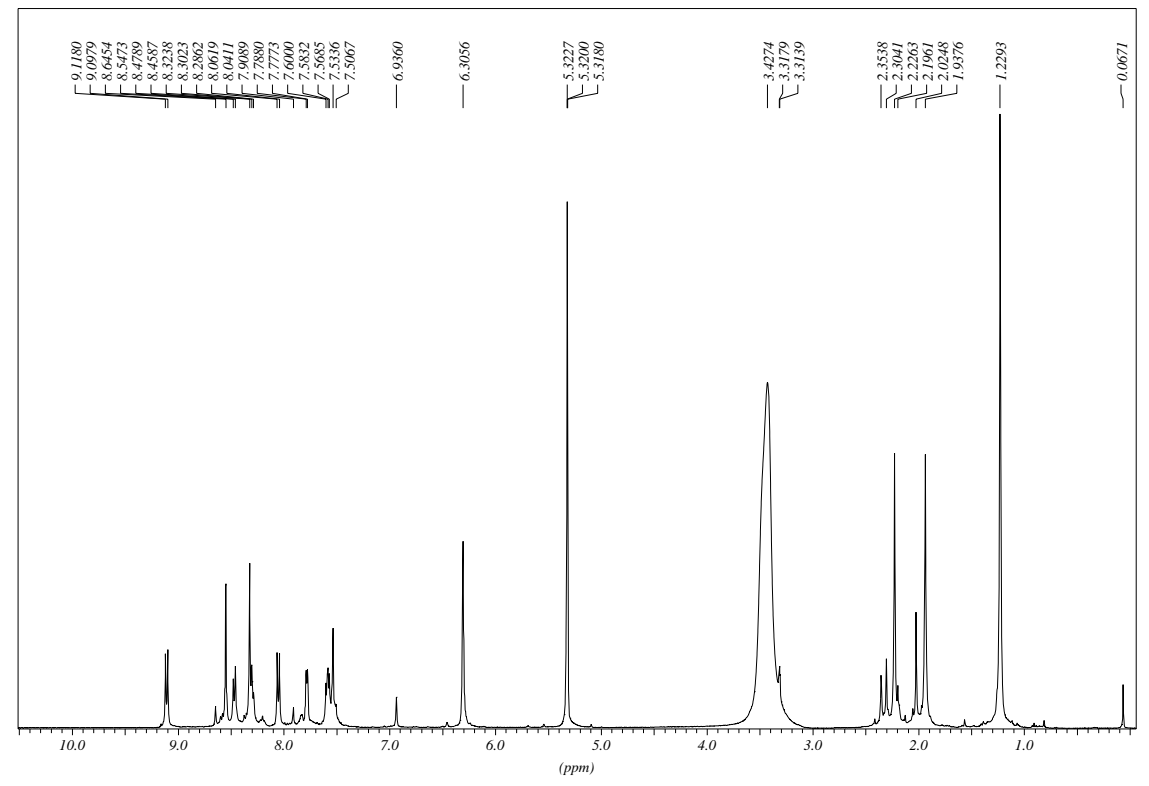

Figure S23. ${ }^{1} \mathrm{H}$ NMR $\left(\mathrm{CD}_{2} \mathrm{Cl}_{2} / \mathrm{CD}_{3} \mathrm{OD}(8: 2)\right.$, $\left.400 \mathrm{MHz}\right)$ spectrum of the dumbbell $\left[\mathrm{Zn}_{2}(\mathbf{1 b})_{2}(2 \mathbf{c})\right](\mathrm{OTf})_{4}$.

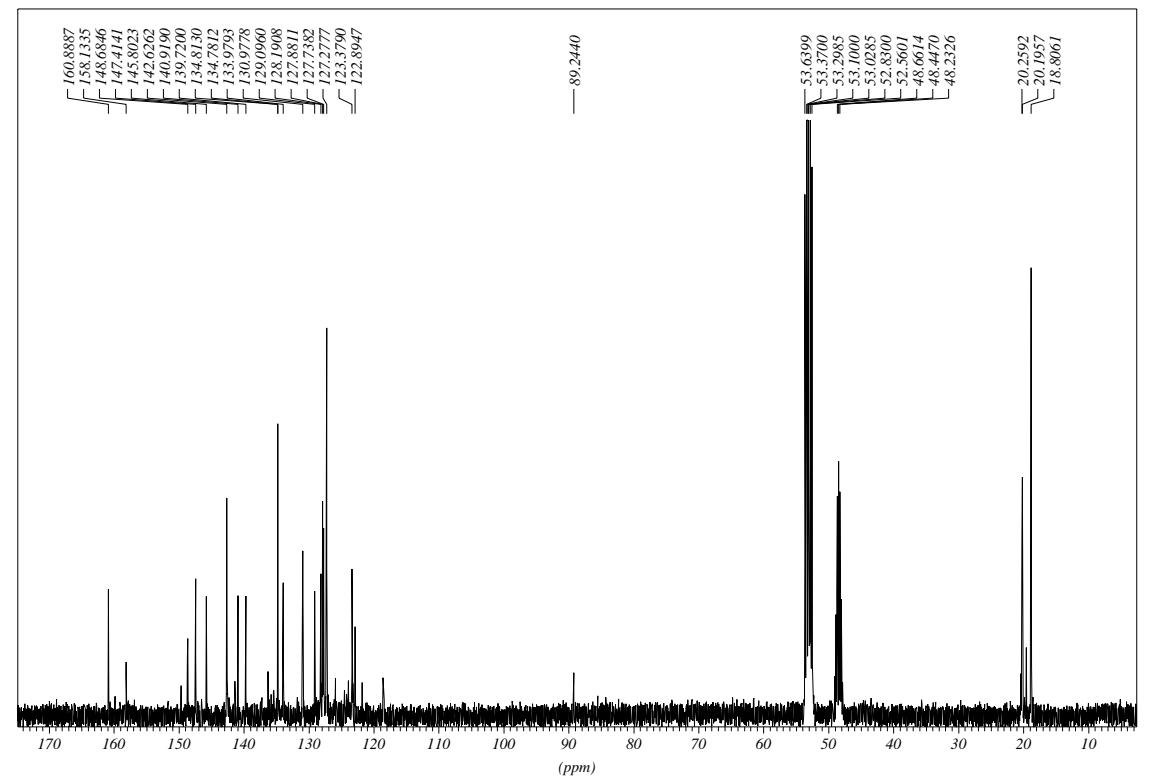

Figure S24. ${ }^{13} \mathrm{C}$ NMR $\left(\mathrm{CD}_{2} \mathrm{Cl}_{2} / \mathrm{CD}_{3} \mathrm{OD}(8: 2), 100 \mathrm{MHz}\right)$ spectrum of the dumbbell $\left[\mathrm{Zn}_{2}(\mathbf{1 b})_{2}(\mathbf{2 c})\right](\mathrm{OTf})_{4}$. 


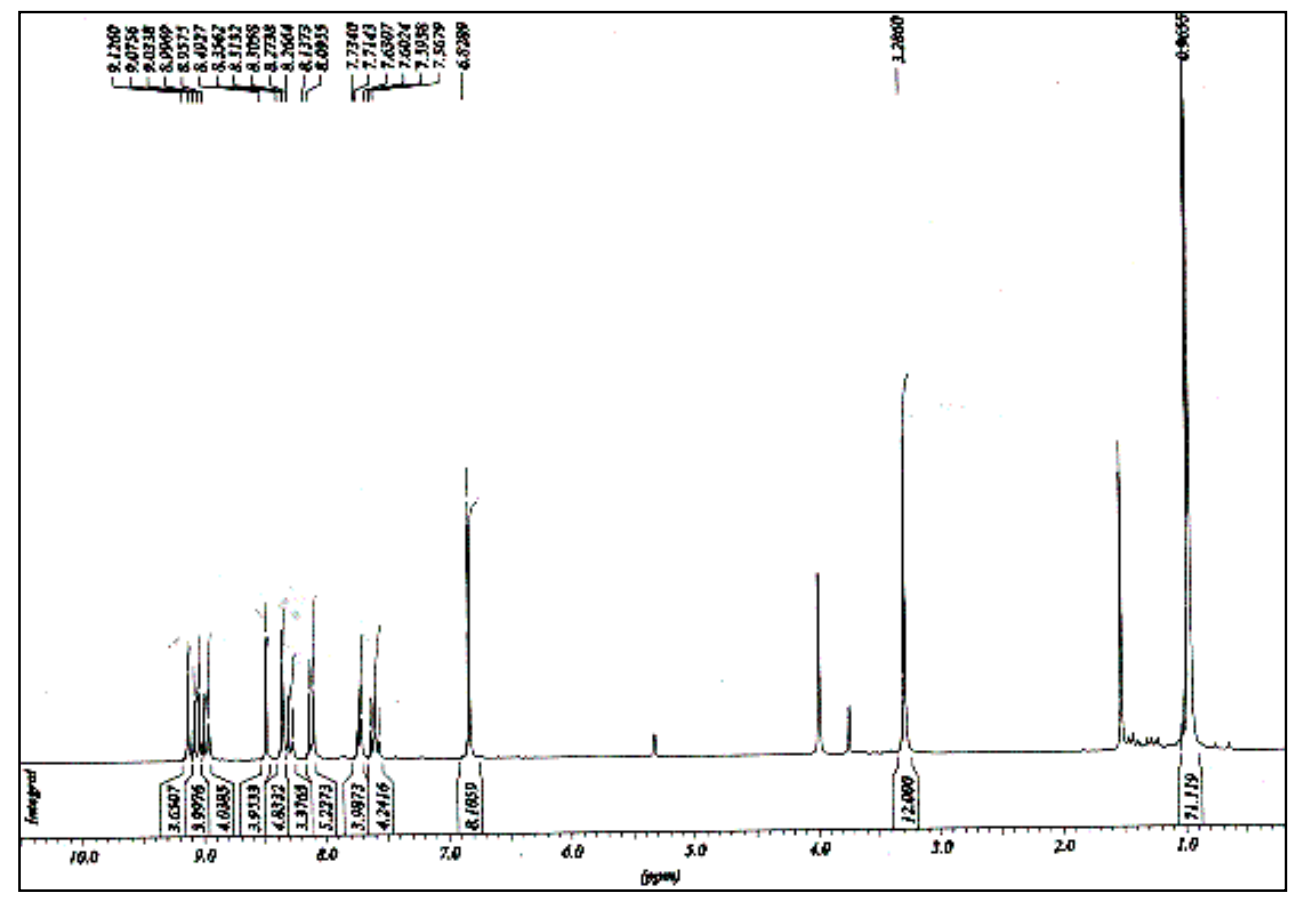

Figure S25. ${ }^{1} \mathrm{H}$ NMR $\left(\mathrm{CD}_{2} \mathrm{Cl}_{2} / \mathrm{CD}_{3} \mathrm{OD}\right.$ (8:2), $\left.200 \mathrm{MHz}\right)$ spectrum of the dumbbell $\left[\mathrm{Zn}_{2}(\mathbf{1 c})_{2}(\mathbf{2 b})\right](\mathrm{OTf})_{4}$.

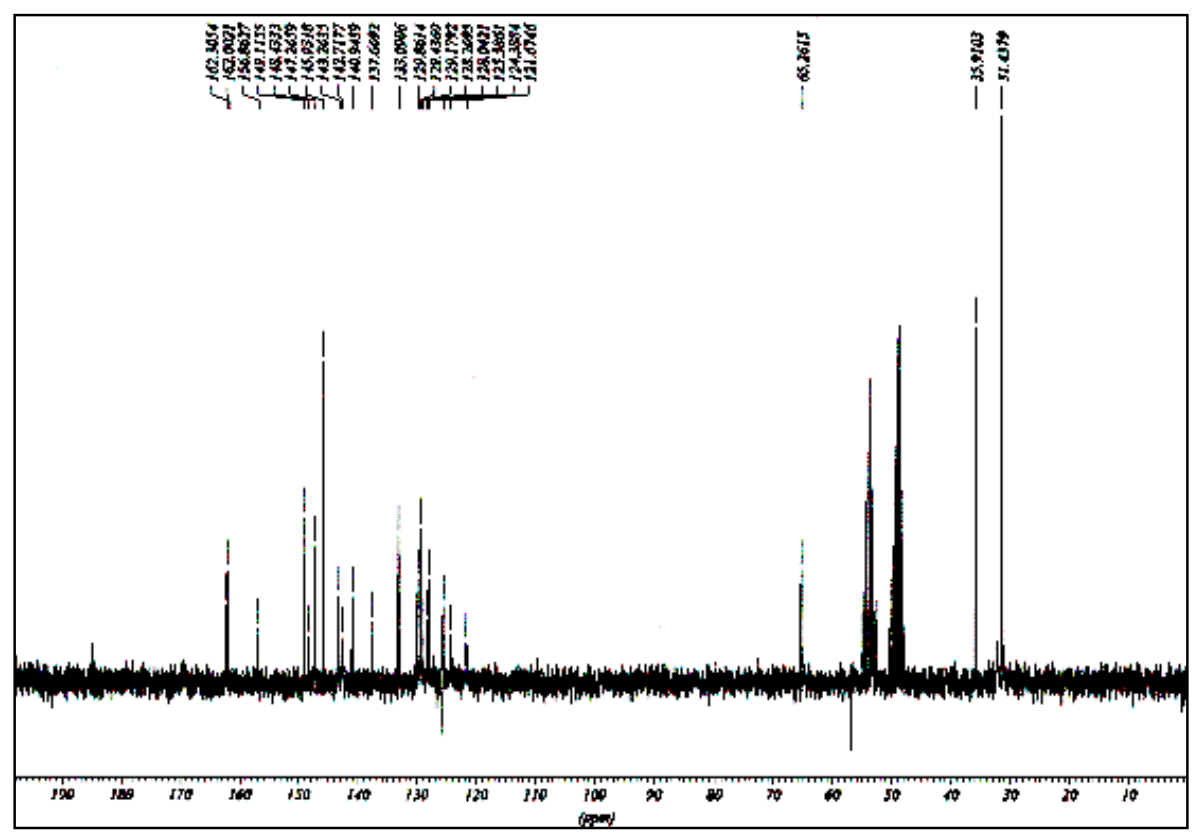

Figure S26. ${ }^{13} \mathrm{C}$ NMR $\left(\mathrm{CD}_{2} \mathrm{Cl}_{2} / \mathrm{CD}_{3} \mathrm{OD}(8: 2), 50 \mathrm{MHz}\right)$ spectrum of the dumbbell $\left[\mathrm{Zn}_{2}(\mathbf{1 c})_{2}(\mathbf{2 b})\right](\mathrm{OTf})_{4}$. 


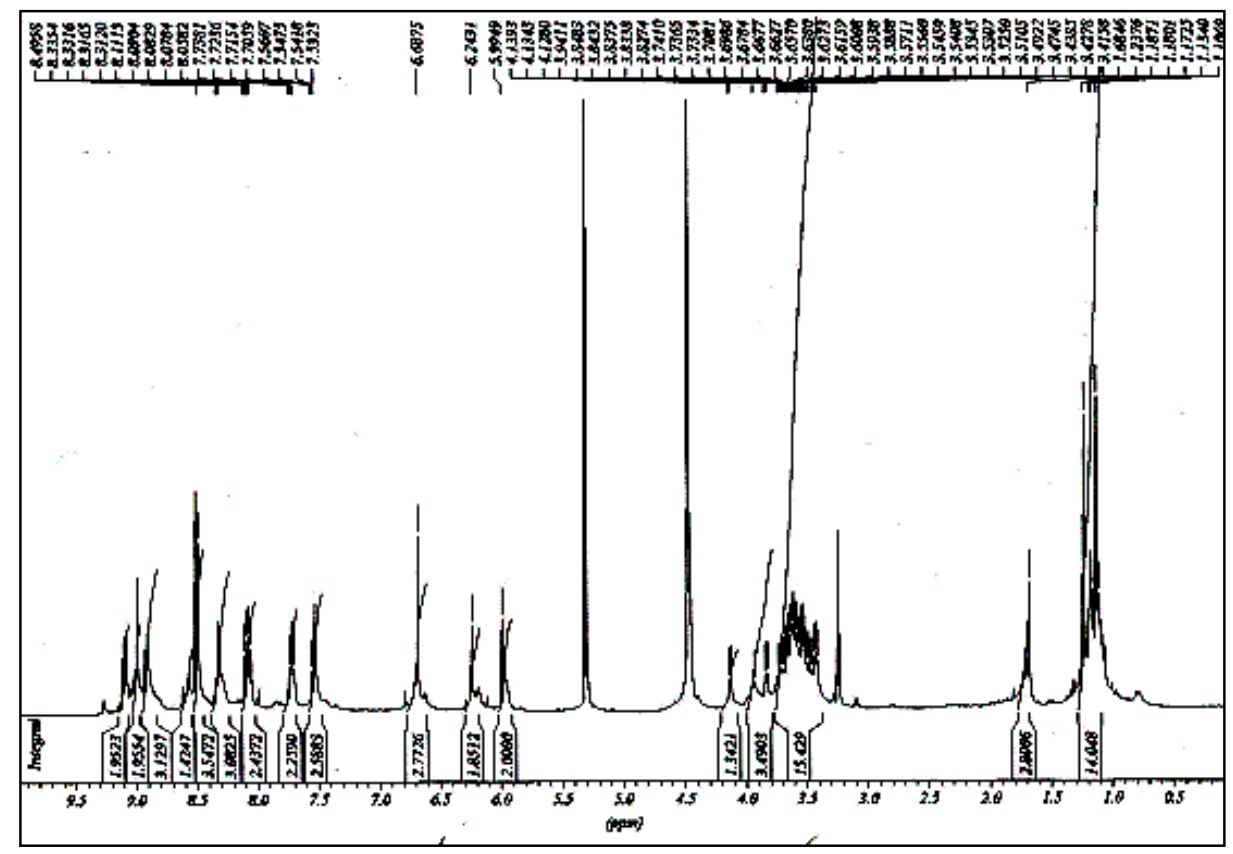

Figure S27. ${ }^{1} \mathrm{H}$ NMR $\left(\mathrm{CD}_{2} \mathrm{Cl}_{2} / \mathrm{CD}_{3} \mathrm{OD}(8: 2), 400 \mathrm{MHz}\right)$ spectrum of the clip $\left[\mathrm{Zn}_{2}(\mathbf{1 d})(\mathbf{2 b})\right]$ $(\mathrm{OTf})_{4}$.

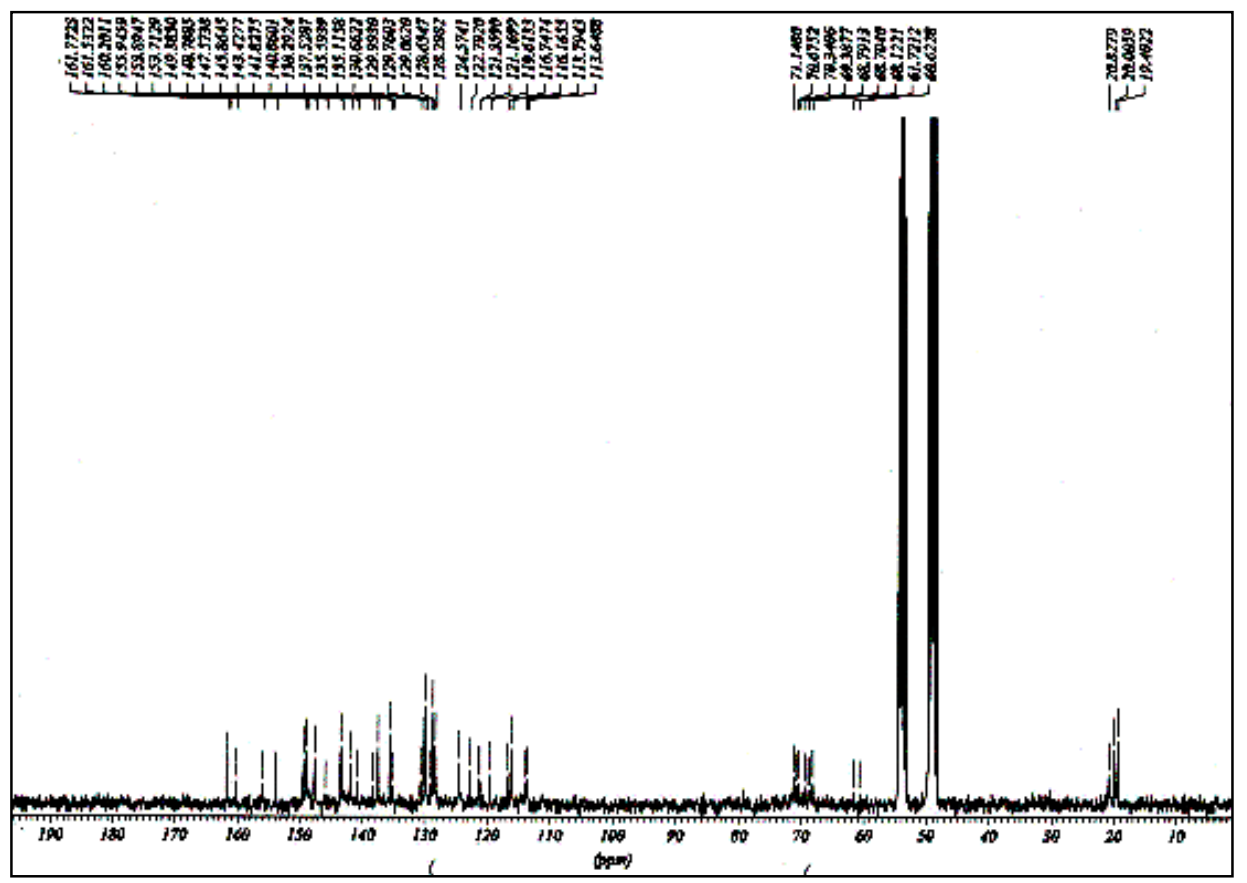

Figure S28. ${ }^{13} \mathrm{C}$ NMR $\left(\mathrm{CD}_{2} \mathrm{Cl}_{2} / \mathrm{CD}_{3} \mathrm{OD}(8: 2), 100 \mathrm{MHz}\right)$ spectrum of the clip $\left[\mathrm{Zn}_{2}\right.$ (1d)(2b)] (OTf) 4 . 


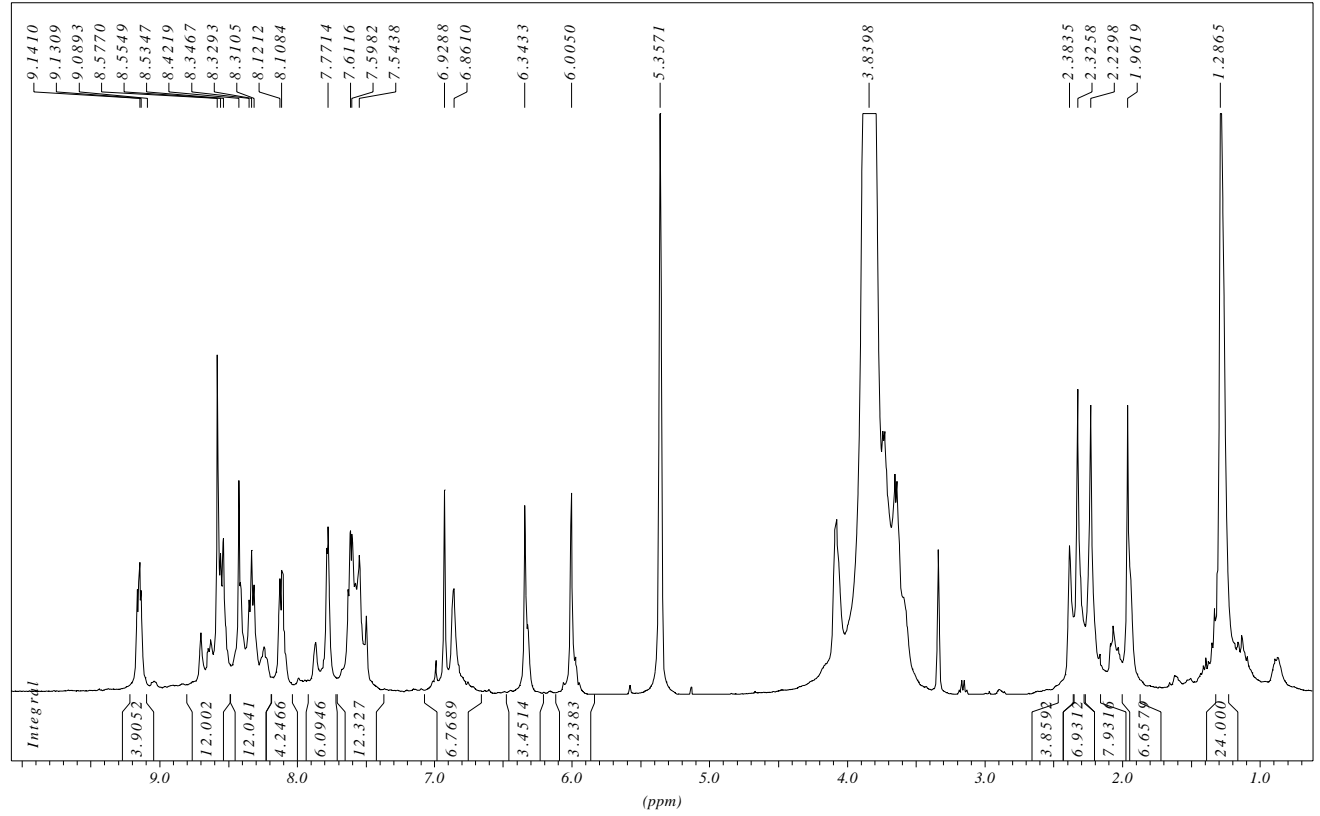

Figure S29. ${ }^{1} \mathrm{H}$ NMR $\left(\mathrm{CD}_{2} \mathrm{Cl}_{2} / \mathrm{CD}_{3} \mathrm{OD}(8: 2), 400 \mathrm{MHz}\right)$ spectrum of the clip $\left[\mathrm{Zn}_{2}\right.$ (1d)(2c)] $(\mathrm{OTf})_{4}$.

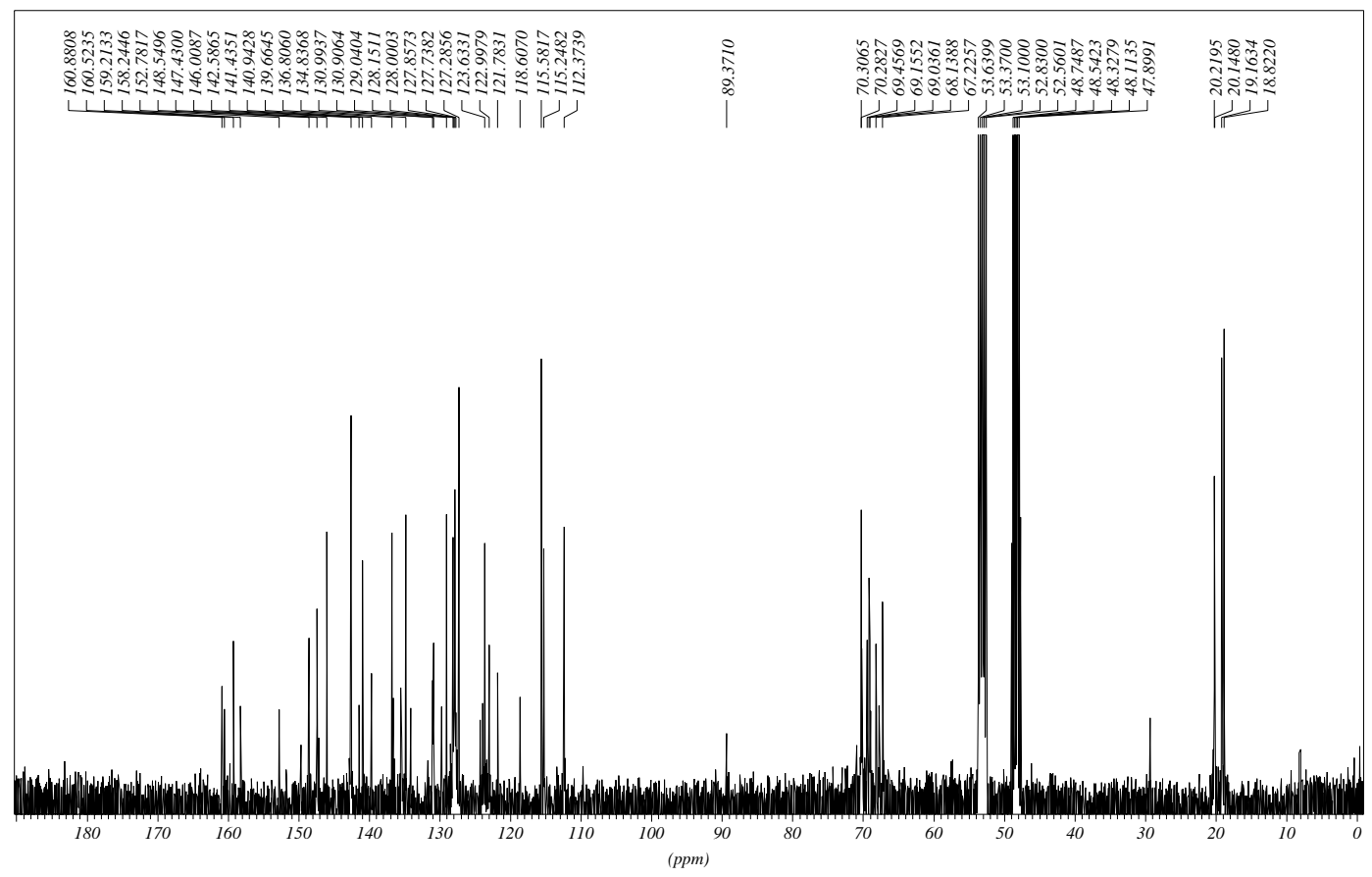

Figure S30. ${ }^{13} \mathrm{C}$ NMR $\left(\mathrm{CD}_{2} \mathrm{Cl}_{2} / \mathrm{CD}_{3} \mathrm{OD}(8: 2), 100 \mathrm{MHz}\right)$ spectrum of the clip $\left[\mathrm{Zn}_{2}(\mathbf{1 d})(2 \mathbf{c})\right]$ $(\mathrm{OTf})_{4}$. 


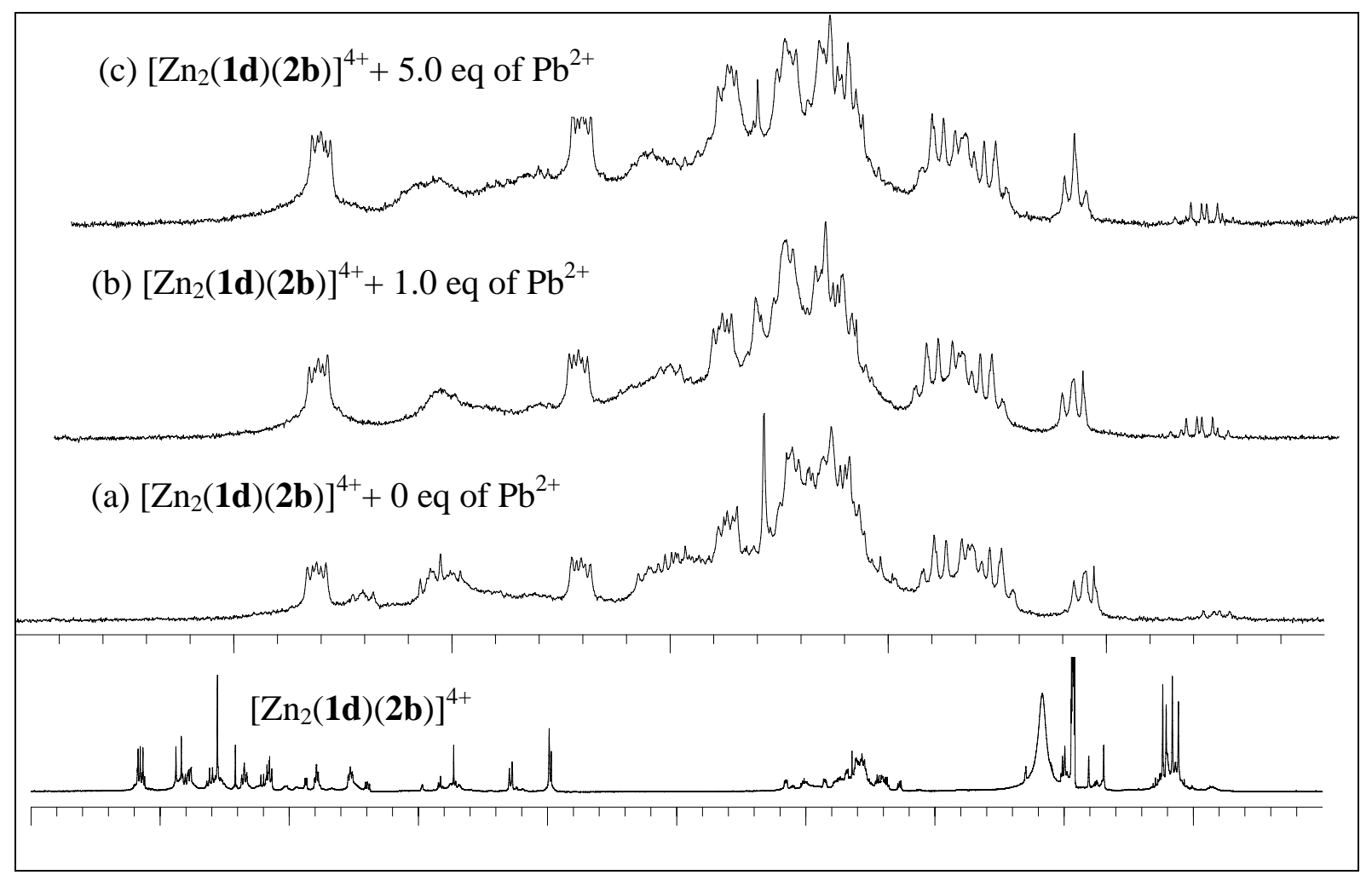

Figure S31. ${ }^{1} \mathrm{H}$ NMR spectra of (a) the complex $\left[\mathrm{Zn}_{2}(\mathbf{1 d})(\mathbf{2 b})\right]^{4+}$ in acetonitrile- $\mathrm{d}_{3}$ and in presence of (b) 1.0 and (c) 5.0 equivalent of $\mathrm{Pb}^{2+}$ (perchlorate salt was used). In the above spectra, the region 3.0 to $4.5 \mathrm{ppm}$ is shown where the change in the signals of the ether linkage upon addition of the metal ion is observed. 


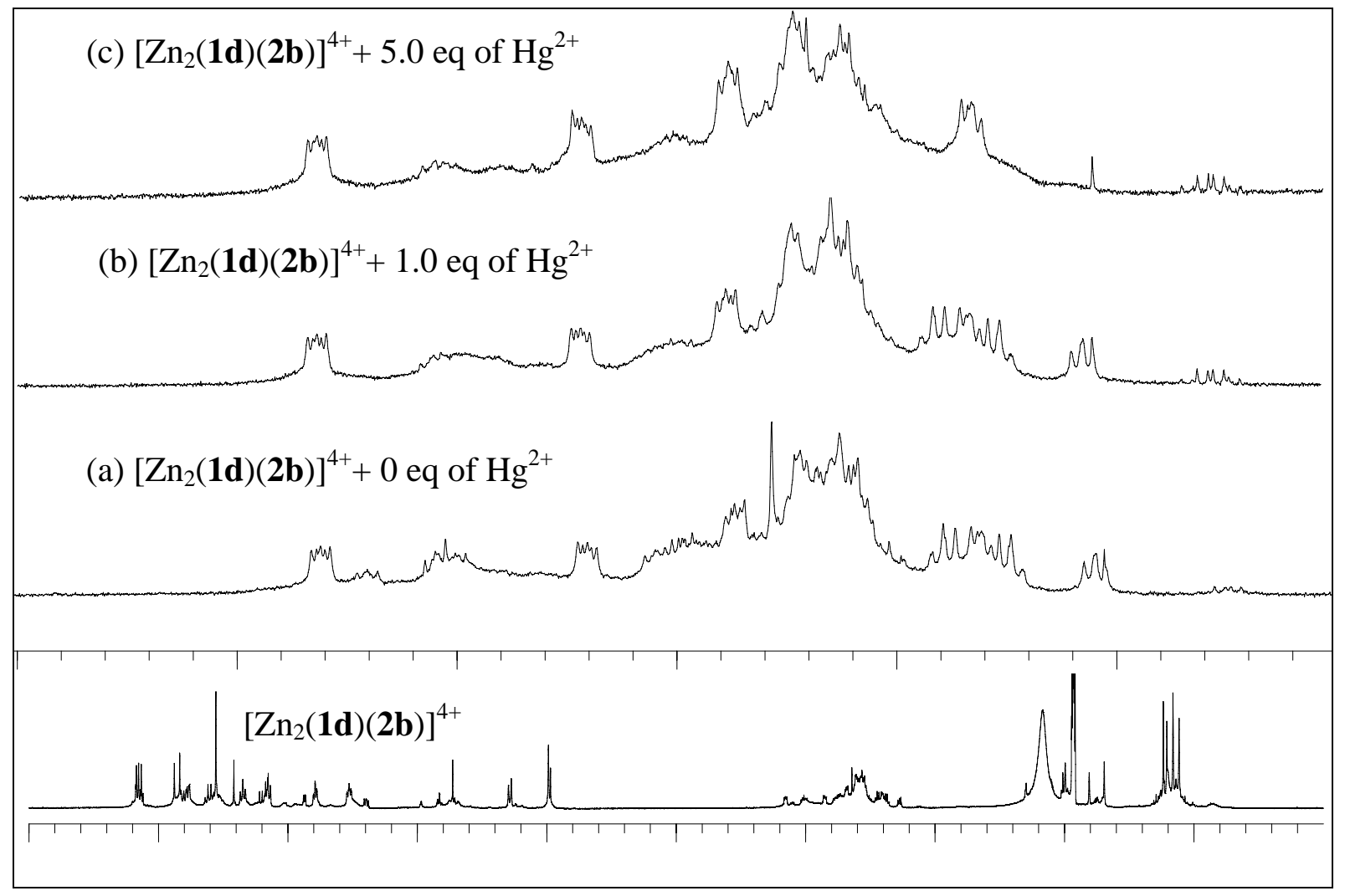

Figure S32. ${ }^{1} \mathrm{H}$ NMR spectra of (a) the complex $\left[\mathrm{Zn}_{2}(\mathbf{1 d})(\mathbf{2 b})\right]^{4+}$ in acetonitrile- $\mathrm{d}_{3}$ and in presence of (b) 1.0 and (c) 5.0 equivalent of $\mathrm{Hg}^{2+}$ (perchlorate salt was used). In the above spectra, the region 3.0 to $4.5 \mathrm{ppm}$ is shown where the change in the signals of the ether linkage upon addition of the metal ion is observed. 